\title{
The Intersubband Approach to Si-based Lasers
}

\author{
Greg Sun \\ University of Massachusetts Boston, Massachusetts, \\ U.S.A.
}

\section{Introduction}

Silicon has been the miracle material for the electronics industry, and for the past twenty years, technology based on $\mathrm{Si}$ microelectronics has been the engine driving the digital revolution. For years, the rapid "Moore's Law" miniaturization of device sizes has yielded an ever-increasing density of fast components integrated on Si chips: but during the time that the feature size was pushed down towards its ultimate physical limits, there has also been a tremendous effort to broaden the reach of Si technology by expanding its functionalities well beyond electronics. $\mathrm{Si}$ is now being increasingly investigated as a platform for building photonic devices. The field of Si photonics has seen impressive growth since early visions in the 1980s and 1990s [1,2]. The huge infrastructure of the global Si electronics industry is expected to benefit the fabrication of highly sophisticated Si photonic devices at costs that are lower than those currently required for compound semiconductors. Furthermore, the Si-based photonic devices make possible the monolithic integration of photonic devices with high speed Si electronics, thereby enabling an oncoming Si-based "optoelectronic revolution".

Among the many photonic devices that make up a complete set of necessary components in Si photonics including light emitters, amplifiers, photodetectors, waveguides, modulators, couplers and switches, the most difficult challenge is the lack of an efficient light source. The reason for this striking absence is that bulk $\mathrm{Si}$ has an indirect band gap where the minimum of the conduction band and the maximum of the valence band do not occur at the same value of crystal momentum in wave vector space (Fig. 1). Since photons have negligible momentum compared with that of electrons, the recombination of an electron-hole pair will not be able to emit a photon without the simultaneous emission or absorption of a phonon in order to conserve the momentum. Such a radiative recombination is a second-order effect occurring with a small probability, which competes with nonradiative processes that take place at much faster rates. As a result, as marvelous as it has been for electronics, bulk Si has not been the material of choice for making light emitting devices including lasers.

Nevertheless, driven by its enormous payoff in technology advancement and commercialization, many research groups around the world have been seeking novel approaches to overcome the intrinsic problem of Si to develop efficient light sources based on $\mathrm{Si}$. One interesting method is to use small $\mathrm{Si}$ nanocrystals dispersed in a dielectric matrix, often times $\mathrm{SiO}_{2}$. Such nano-scaled $\mathrm{Si}$ clusters are naturally formed by the thermal annealing of a Si-rich oxide thin film. Silicon nanocrystals situated in a much wider band gap $\mathrm{SiO}_{2} \mathrm{can}$ effectively localize electrons with quantum confinement, which improves the radiative recombination probability, shifts the emission spectrum toward shorter wavelengths, and 


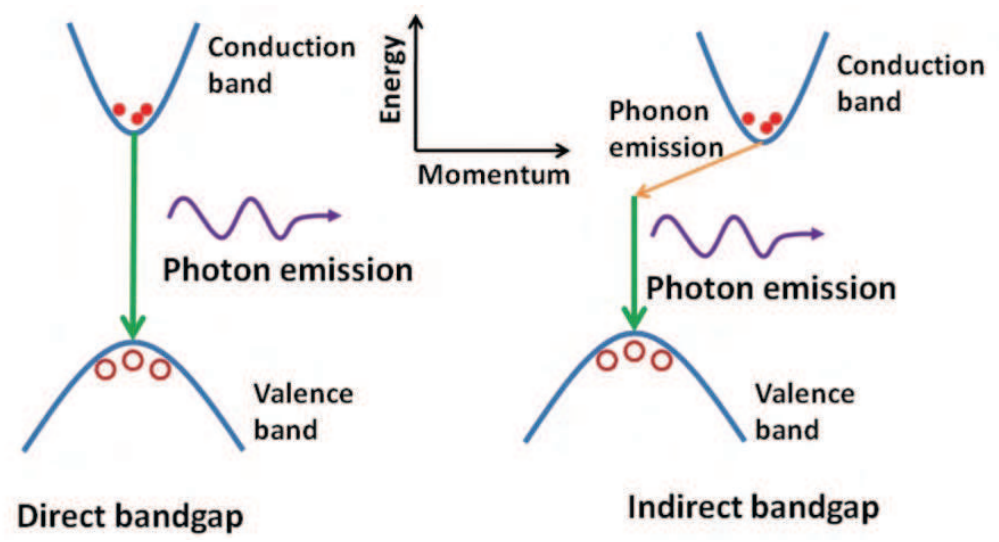

Fig. 1. Illustration of a photon emission process in (a) the direct and (b) the indirect band gap semiconductors.

decreases the free carrier absorption. Optical gain and stimulated emission have been observed from these Si nanocrystals by both optical pumping $[3,4]$ and electrical injection [5], but the origin of the observed optical gain has not been fully understood as the experiments were not always reproducible - results were sensitive to the methods by which the samples were prepared. In addition, before Si-nanocrystal based lasers can be demonstrated, the active medium has to be immersed in a tightly confined optical waveguide or cavity.

Another approach is motivated by the light amplification in Er-doped optical fibers that utilize the radiative transitions in $\mathrm{Er}$ ions $\left(\mathrm{Er}^{3+}\right)[6]$. By incorporating $\mathrm{Er}^{3+}$ in $\mathrm{Si}$, these ions can be excited by energy transfer from electrically injected electron-hole pairs in Si and will subsequently relax by emitting photons at the telecommunication wavelength of $1.55 \mu \mathrm{m}$. However, the concentration of $\mathrm{Er}^{3+}$ ions that can be doped in $\mathrm{Si}$ is relatively low and there is a significant energy back-transfer from the $\mathrm{Er}^{3+}$ ions to the $\mathrm{Si}$ host due to the resonance with a defect level in Si. As a result, both efficiency and maximum power output have been extremely low $[7,8]$. To reduce the back transfer of energy, $\mathrm{SiO}_{2}$ with an enlarged band gap has been proposed as host to remove the resonance between the defect and the $\mathrm{Er}^{3+}$ energy levels [9]. Once again, $\mathrm{Si}$-rich oxide is employed to form $\mathrm{Si}$ nanocrystals in close proximity to $\mathrm{Er}^{3+}$ ions. The idea is to excite $\mathrm{Er}^{3+}$ ions with the energy transfer from the nearby $\mathrm{Si}$ nanocrystals. Light emitting diodes (LEDs) with efficiencies of about $10 \%$ have been demonstrated [10] on par with commercial devices made of GaAs, but with power output only in tens of $\mu \mathrm{W}$. While there have been proposals to develop lasers using doped Er in Sibased dielectric, the goal remains elusive.

The only approach so far that has led to the demonstration of lasing in Si exploited the effect of stimulated Raman scattering [11-13], analogous to that produced in fiber Raman amplifiers. With both the optical pumping and the Raman scattering below the band gap of $\mathrm{Si}$, the indirectness of the Si band gap becomes irrelevant. Depending on whether it is a Stokes or anti-Stokes process, the Raman scattering either emits or absorbs an optical phonon. Such a nonlinear process requires optical pumping at very high intensities $\left(\sim 100 \mathrm{MW} / \mathrm{cm}^{2}\right)$ and the device lengths $(\sim \mathrm{cm})$ are too large to be integrated with other photonic and electronic devices in any type of Si VLSI-type circuit [14]. 
Meanwhile, the search for laser devices that can be integrated on Si chips has gone well beyond the monolithic approach to seek solutions using hybrid integration of III-V compounds with Si. A laser with an AlGaInAs quantum well (QW) active region bonded to a silicon waveguide cavity was demonstrated [15]. This fabrication technique allows for the optical waveguide to be defined by the CMOS compatible Si process while the optical gain is provided by III-V materials. Rare-earth doped visible-wavelength GaN lasers fabricated on Si substrates are also potentially compatible with the Si CMOS process [16]. Another effort produced InGaAs quantum dot lasers deposited directly on Si substrates with a thin GaAs buffer layer [17]. Although these hybrid approaches offer important alternatives, they do not represent the ultimate achievement of Si-based lasers monolithically integrated with Si electronics.

While progress is being made along these lines and debates continue about which method offers the best promise, yet another approach emerged that has received a great deal of attention in the past decade-an approach in which the lasing mechanism is based on intersubband transitions (ISTs) in semiconductor QWs. Such transitions take place between quantum confined states (subbands) of conduction or valence bands and do not cross the semiconductor band gap. Since carriers remain in the same energy band (either conduction or valence), optical transitions are always direct in momentum space rendering the indirectness of the Si band gap irrelevant. Developing lasers using ISTs therefore provides a promising alternative that completely circumvents the issue of indirectness in the Si band gap. In addition, this type of laser can be conveniently designed to employ electrical pumping - the so-called quantum cascade laser (QCL). The pursuit of Si-based QCLs might turn out to be a viable path to achieving electrically pumped Si-based coherent emitters that are suitable for monolithic integration with Si photonic and electronic devices.

In this chapter, lasing processes based on ISTs in QWs are explained by drawing a comparison to conventional band-to-band lasers. Approaches and results towards SiGe QCLs using ISTs in the valence band are overviewed, and the challenges and limitations of the SiGe valence-band QCLs are discussed with respect to materials and structures. In addition, ideas are proposed to develop conduction-band QCLs, among them a novel QCL structure that expands the material combination to SiGeSn. This is described in detail as a way to potentially overcome the difficulties that are encountered in the development of SiGe QCLs.

\section{Lasers based on intersubband transitions}

Research on quantum confined structures including semiconductor QWs and superlattices (SLs) was pioneered by Esaki and Tsu in 1970 [18]. Since then confined structures have been developed as the building blocks for a majority of modern-day semiconductor optoelectronic devices. QWs are formed by depositing a narrower band gap semiconductor with a layer thickness thinner than the deBroglie wavelength of the electron $(\sim 10 \mathrm{~nm})$ between two wider band gap semiconductors (Fig. 2(a)). The one-dimensional quantum confinement leads to quantized states (subbands) in the direction of growth $z$ within both conduction and valence bands. The energy position of each subband depends on the band offset $\left(\Delta E_{C}, \Delta E_{V}\right)$ and the effective mass of the carrier. In directions perpendicular to $z$ (inplane), the carriers are unconfined and can thus propagate with an in-plane wave vector $\boldsymbol{k}$ which gives an energy dispersion for each subband. (Fig. 2(b)) 


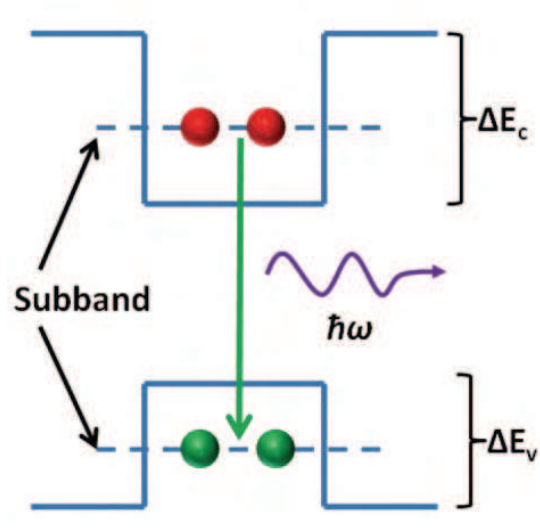

(a)

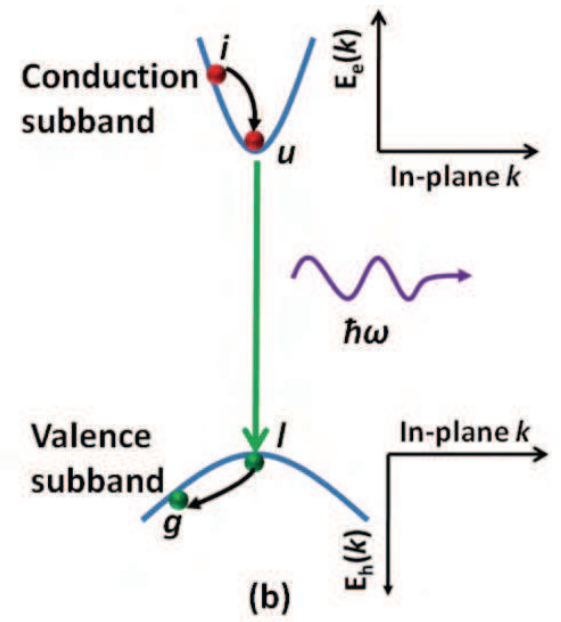

(b)

Fig. 2. Illustration of (a) conduction and valence subband formations in a semiconductor QW and (b) in-plane subband dispersions with optical transitions between conduction and valence subbands.

Obviously, if the band offset is large enough, there could be multiple subbands present within either conduction or valence band as shown in Fig. 3 where two subbands are confined within the conduction band. The electron wavefunctions (Fig. 3(a)) and energy dispersions (Fig. 3(b)) are illustrated for the two subbands. The concept of ISTs refers to the physical process of a carrier transition between these subbands within either the conduction or valence band as illustrated in Fig. 3. Carriers originally occupying a higher energy subband can make a radiative transition to a lower subband by emitting a photon. Coherent sources utilizing this type of transition as the origin of light emission are called intersubband lasers.

The original idea of creating light sources based on ISTs was proposed by Kazarinov and Suris [19] in 1971, but the first QCL was not demonstrated until 1994 by a group led by Capasso at Bell Laboratories [20]. In comparison with the conventional band-to-band lasers, lasers based on ISTs require much more complex design of the active region which consists of carefully arranged multiple QWs (MQWs). The reason for added complexity can be appreciated by comparing the very different band dispersions that are involved in these two types of lasers. In a conventional band-to-band laser, it appears that the laser states consist of two broad bands. But a closer look at the conduction and valence band dispersions (Fig. 2(b)) reveals a familiar four-level scheme where in addition to the upper laser states $|u\rangle$, located near the bottom of the conduction band and the lower laser states $\mid l>$, near the top of the valence band, there are two other participating states - intermediate states $|i\rangle$, and ground states $|g\rangle$. The pumping process (either injection or optical) places electrons into the intermediate states, $|i\rangle$, from which they quickly relax toward the upper laser states $\mid u>$ by inelastic scattering intraband processes. This process is very fast, occurring on a subpico-second scale. But once they reach states $|u\rangle$, they tend to stay there for a much longer time determined by the band-to-band recombination rate which is on the order of nanoseconds. Electrons that went through lasing transitions to the lower laser states $|l\rangle$ will quickly scatter into the lower energy states of the valence band - ground states $\mid g>--$ 
by the same fast inelastic intraband processes. (A more conventional way to look at this is the relaxation of holes toward the top of the valence band.) The population inversion between $\mid u>$ and $|l\rangle$ is therefore established mostly by the fundamental difference between the processes determining the lifetimes of upper and lower laser states. As a result, the lasing threshold can be reached when the whole population of the upper conduction band is only a tiny fraction of that of the lower valence band.

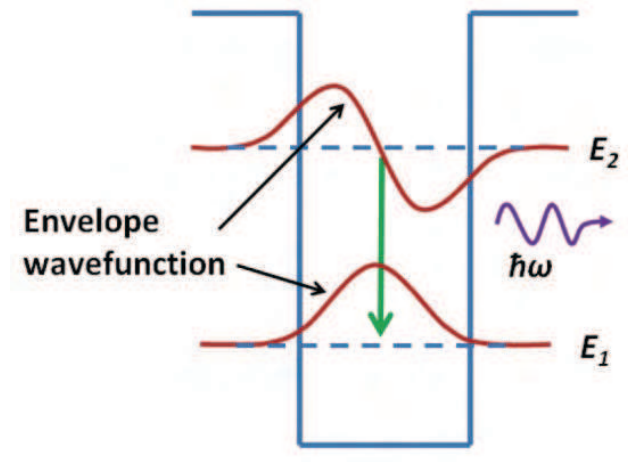

(a)

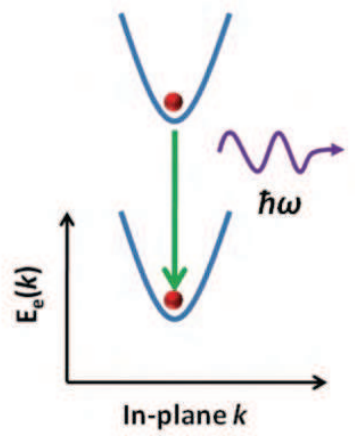

(b)

Fig. 3. (a) Two subbands formed within the conduction band confined in a QW and their election envelope functions, (b) in-plane energy dispersions of the two subbands. Radiative intersubband transition between the two subbands is highlighted.

Let us now turn our attention to the intersubband transition shown in Fig. 3(b). The in-plane dispersions of the upper $\mid u>$ and lower $|l\rangle$ conduction subbands are almost identical when the band nonparabolicity can be neglected. For all practical purposes they can be considered as two discrete levels. Then, in order to achieve population inversion it is necessary to have the whole population of the upper subband exceed that of the lower subband. For this reason, a three- or four-subband scheme becomes necessary to reach the lasing threshold. Even then, since the relaxation rates between different subbands are determined by the same intraband processes, a complex multiple QW structure needs to be designed to engineer the lifetimes of involved subbands.

Still, intersubband lasers offer advantages in areas where the conventional band-to-band lasers simply cannot compete. In band-to-band lasers, lasing wavelengths are mostly determined by the intrinsic band gap of the semiconductors. There is very little room for tuning, accomplished by varying the structural parameters such as strain, alloy composition, and layer thickness. Especially for those applications in the mid-IR to far-IR range, there are no suitable semiconductors with the appropriate band gaps from which such lasers can be made. With the intersubband transitions, we are no longer limited by the availability of semiconductor materials to produce lasers in this long wavelength region. In addition, for ISTs between conduction subbands with parallel band dispersions, the intersubband lasers should therefore have a much narrower gain spectrum in comparison to the band-to-band lasers in which conduction and valence bands have opposite band curvatures.

A practical design that featured a four-level intersubband laser pumped optically was proposed by Sun and Khurgin [21,22] in the early 1990s. This work laid out a comprehensive 
analysis of various intersubband processes that affect the lasing operation including scattering mechanisms that determine subband lifetimes, conditions for population inversion between two subbands, band engineering to achieve it, and optical gain sufficient to compensate for losses under realistic pumping intensity. The QCLs developed soon thereafter significantly expanded the design in order to accommodate electrical pumping by implementing a rather elaborate scheme of current injection with the use of a chirped SL as the injector region placed in between the active regions (Fig. 4). The QCL has a periodic structure with each period consisting of an active and an injector region. Both active and injector regions are composed of MQWs. By choosing combinations of layer thicknesses and material compositions, three subband levels with proper energy separations and wave function overlaps are obtained in the active region. The injector region, on the other hand, is designed with a sequence of QWs having decreasing well widths (chirped SL) such that they form a miniband under an electric bias that facilitates electron transport. The basic operating principle of a QCL is illustrated in Fig. 4. Electrons are first injected through a barrier into subband 3 (upper laser state) of the active region, they then undergo lasing transitions to subband 2 (lower laser state) by emitting photons, followed by fast depopulation into subband 1 via nonradiative processes. These electrons are subsequently transported through the injector region into the next active region where they repeat the process in a cascading manner, typically 20 to 100 times.

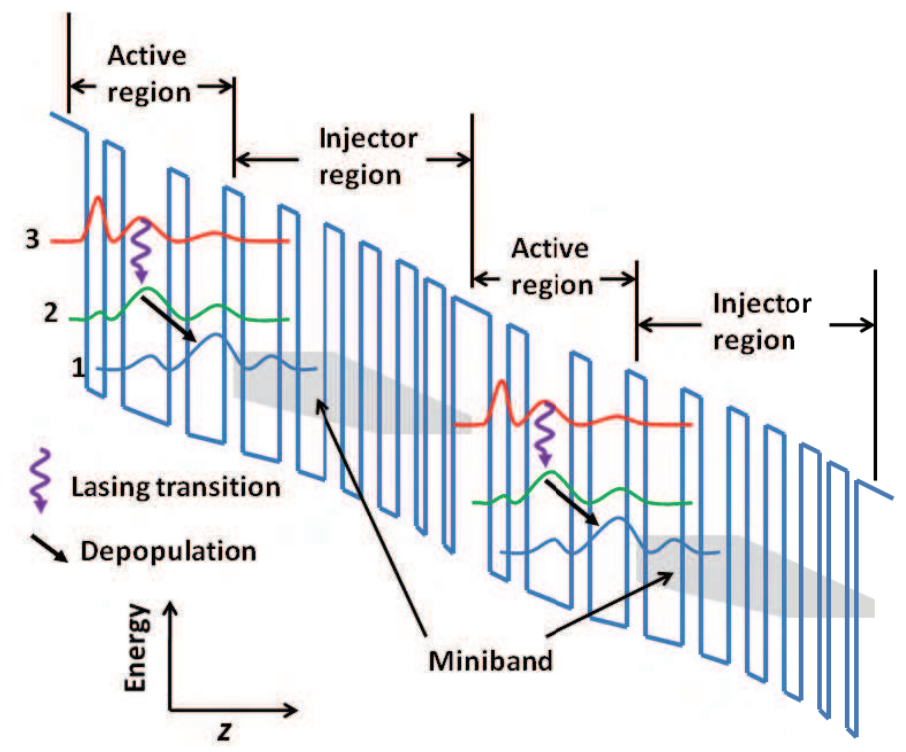

Fig. 4. Schematic band diagram of two periods of a QCL structure with each period consisting of an active and an injector region. Lasing transitions are between the states 3 and 2 in the active regions with rapid depopulation of lower state 2 into state 1 which couples strongly with the minibands formed in injector regions that transport carriers to state 3 in the next period. The magnitude-squared wavefunctions for the three subbands in active regions are illustrated. 
Advances of QCLs since the first demonstration have resulted in dramatic performance improvement in spectral range, power and temperature. They have become the dominant mid-IR semiconductor laser sources covering the spectral range of $3<\lambda<25 \mu \mathrm{m}$ [23-25], many of them operating in the continuous-wave mode at room temperature with peak power reaching a few watts [26,27]. Meanwhile, QCLs have also penetrated deep into the $\mathrm{THz}$ regime loosely defined as the spectral region $100 \mathrm{GHz}<f<10 \mathrm{THz}$ or $30<\lambda<3000$ $\mu \mathrm{m}$, bridging the gap between the far-IR and $\mathrm{GHz}$ microwaves. At present, spectral coverage from $0.84-5.0 \mathrm{THz}$ has been demonstrated with operation in either the pulsed or continuous-wave mode at temperatures well above 100K [28].

\section{Intersubband theory}

In order to better explain the design considerations of intersubband lasers, it is necessary to introduce some basic physics that underlies the formation of subbands in QWs and their associated intersubband processes. The calculation procedures described here follows the envelope function approach based on the effective-mass approximation [29]. The $\boldsymbol{k} \cdot \boldsymbol{p}$ method [30] was outlined to obtain in-plane subband dispersions in the valence band. Optical gain for transitions between subbands in conduction and valence bands is derived. Various scattering mechanisms that determine the subband lifetimes are discussed with an emphasis on the carrier-phonon scattering processes.

\subsection{Subbands and dispersions}

Let us treat the conduction subbands first. It is well known in bulk material that near the band edge, the band dispersion with an isotropic effective mass follows a parabolic relationship. In a QW structure, along the in-plane direction $\left(\boldsymbol{k}=k_{x} \hat{x}+k_{y} \hat{y}\right)$ where electrons are unconfined, such curvature is preserved for a given subband $i$, assuming the nonparabolicity that describes the energy-dependent effective mass $m_{e}^{*}$ can be neglected,

$$
E_{i, \boldsymbol{k}}=E_{i}+\frac{\hbar^{2} k^{2}}{2 m_{e}^{*}}
$$

where $\hbar$ is the Planck constant and $E_{i}$ is the minimum energy of subband $i$ in a QW structure. This minimum energy can be calculated as one of the eigen values of the Schrödinger equation along the growth direction $z$,

$$
\left[-\frac{\hbar^{2}}{2} \frac{d}{d z} \frac{1}{m_{e}^{*}(z)} \frac{d}{d z}+V_{c}(z)\right] \varphi_{i}(z)=E_{i} \varphi_{i}(z)
$$

where the $z$-dependence of $m_{e}^{*}$ allows for different effective masses in different layers and $V_{c}(z)$ represents the conduction band edge along the growth direction $z$, . The envelope function of subband $i, \varphi_{i}(z)$, together with the electron Bloch function $u_{e}(\boldsymbol{R})$ and the plane wave $e^{j k \cdot r}$, gives the electron wavefunction in the QW structure as

$$
\Phi_{i}(\boldsymbol{r}, z)=\varphi_{i}(z) u_{e}(\boldsymbol{R}) e^{j \boldsymbol{k} \cdot \boldsymbol{r}}
$$

where the position vector is decomposed into in-plane and growth directions $\boldsymbol{R}=\boldsymbol{r}+z \hat{\mathbf{z}}$. Since we are treating electron subbands, the Bloch function is approximately the same for all subbands and all $\boldsymbol{k}$-vectors. The electron envelope function can be given as a combination of 
the forward and backward propagations in a given region $l$ of the QW structure (either a QW or a barrier region), $d_{l}<z<d_{l+1}$

$$
\varphi_{i}(z)=A_{l} e^{j k_{z}\left(z-d_{l}\right)}+B_{l} e^{-j k_{z}\left(z-d_{l}\right)}
$$

where $A_{l}$ and $B_{l}$ are constants that need to be fixed with the continuity conditions at each of the interfaces $z=d_{l}$,

$$
\varphi_{i}(z) \text { and } \frac{1}{m_{e}^{*}(z)} \frac{d \varphi_{i}(z)}{d z} \text { continous }
$$

in conjunction with the relationship between the subband minimum energy $E_{i}$ and the quantized wave vector $k_{z}$ in the $z$-direction

$$
E_{i}=\frac{\hbar^{2} k_{z}^{2}}{2 m_{e}^{*}(z)}+V_{c}(z)
$$

where $k_{z}$ assumes either real or imaginary value depending on $E_{i}-V_{c}(z)$. The continuity conditions in Eq.(5) ensure continuous electron distribution and conservation of electron current across the interface.

In the presence of an electric field $\mathbb{E}$ applied in the $z$-direction, the potential term $V_{c}(z)$ in the Schrödinger equation Eq.(2) becomes tilted along the $z$-direction according to $-e \mathbb{E} z$. If the Coulomb effect due to the distribution of electrons in the subband needs to be taken into consideration, then the potential in region $l$ of the QW structure with the conduction band edge $V_{c, l}$ should be modified as

$$
V_{c}(z)=V_{c, l}-e \mathbb{E} z-e \phi(z)
$$

where $e \phi(z)$ takes into account the potential due to electron distributions in all subbands and can be obtained by solving the Poisson equation

$$
\frac{\partial^{2}}{\partial z^{2}} \phi(z)=-\frac{e}{\varepsilon_{0} \varepsilon(z)}\left[\sum_{i} n_{i}\left|\varphi_{i}(z)\right|^{2}-N_{d}(z)\right]
$$

consistently with Eq.(2), where $e$ is the charge of a free electron, $\varepsilon_{0}$ is the permittivity of free space, $\varepsilon(z)$ is the $z$-dependent dielectric constant of the QW structure, $n_{i}$ is the electron density of subband $i$, and $N_{d}(z)$ is the n-type doping profile in the structure.

In comparison with the conduction band, the situation in the valence band is far more complex mostly because of the interactions between subbands of different effective masses that produce strong nonparabolicity. The in-plane dispersion of valence subbands and their associated envelope functions can be obtained in the framework of the effective mass approximation by applying the $\boldsymbol{k} \cdot \boldsymbol{p}$ theory [30] to QWs [31] where, in the most general treatment, an $8 \times 8$ Hamiltonian matrix is employed to describe the interactions between the conduction, heavy-hole ( $\mathrm{HH})$, light-hole ( $\mathrm{LH})$, and spin-orbit split off (SO) bands. Often times, for semiconductors in which the conduction band is separated far in energy from the valence band, the coupling of the conduction band can be ignored. For the group-IV semiconductors $\mathrm{Si}$ and Ge with indirect band gaps, this approximation is particularly adequate. In those structures where there is little strain such as GaAs/AlGaAs, the SO band coupling can also be ignored. The $8 \times 8$ Hamiltonian matrix can then be reduced to a $4 \times 4$ matrix. But for systems with appreciable lattice mismatch, strain induces strong coupling 
between $\mathrm{LH}$ and SO bands. For the SiGe system with a large lattice mismatch, the SO band should be included and a $6 \times 6$ matrix Hamiltonian equation needs to be solved to come up with the dispersion relations and envelope functions. Such a $6 \times 6$ matrix Hamiltonian equation can be solved exactly in multiple QW structures under the bias of an electric field. A procedure based on the Luttinger-Kohn Hamiltonian [32,33] is outlined as follows.

The $6 \times 6$ Luttinger-Kohn Hamiltonian matrix including the uniaxial stress along (001) is given in the $\mathrm{HH}\left(\mid \frac{3}{2}, \pm \frac{3}{2}>\right), \mathrm{LH}\left(\mid \frac{3}{2}, \pm \frac{1}{2}>\right)$, and $\mathrm{SO}\left(\mid \frac{1}{2}, \pm \frac{1}{2}>\right)$ Bloch function space as

$$
H=-\left[\begin{array}{ccccccc}
\mid \frac{3}{2}, \frac{3}{2}> & \left|\frac{3}{2}, \frac{1}{2}\right\rangle\left|\frac{3}{2},-\frac{1}{2}>\right| \frac{3}{2},-\frac{3}{2}> & \mid \frac{1}{2}, \frac{1}{2}> & \left|\frac{1}{2},-\frac{1}{2}\right\rangle \\
P+Q & -S & R & 0 & -\frac{1}{\sqrt{2}} S & \sqrt{2} R \\
-S^{\dagger} & P-Q & 0 & R & -\sqrt{2} Q & \sqrt{\frac{3}{2} S} \\
R^{\dagger} & 0 & P-Q & S & \sqrt{\frac{3}{2} S^{\dagger}} & \sqrt{2} Q \\
0 & R^{\dagger} & S^{\dagger} & P+Q & -\sqrt{2} R^{\dagger} & -\frac{1}{\sqrt{2}} S^{\dagger} \\
-\frac{1}{\sqrt{2}} S^{\dagger} & -\sqrt{2} Q & \sqrt{\frac{3}{2} S} & -\sqrt{2} R & P+\Delta & 0 \\
\sqrt{2} R^{\dagger} & \sqrt{\frac{3}{2}} S^{\dagger} & \sqrt{2} Q & -\frac{1}{\sqrt{2}} S & 0 & P+\Delta
\end{array}\right]+V_{v}(z)
$$

where $V_{v}(z)$ is the valence band edge profile (degenerate for $\mathrm{HH}$ and $\mathrm{LH}$ bands) of the QW structure,

$$
\begin{gathered}
P=\frac{\hbar^{2}}{2 m_{0}} \gamma_{1}\left(k_{x}^{2}+k_{y}^{2}+k_{z}^{2}\right)-a_{v}\left(\epsilon_{x x}+\epsilon_{y y}+\epsilon_{z z}\right) \\
Q=\frac{\hbar^{2}}{2 m_{0}} \gamma_{2}\left(k_{x}^{2}+k_{y}^{2}-2 k_{z}^{2}\right)-\frac{b}{2}\left(\epsilon_{x x}+\epsilon_{y y}-2 \epsilon_{z z}\right) \\
S=\frac{\hbar^{2}}{2 m_{0}} 2 \sqrt{3} \gamma_{3}\left(k_{x}-j k_{y}\right) k_{z} \\
R=\frac{\hbar^{2}}{2 m_{0}} \sqrt{3}\left[-\gamma_{2}\left(k_{x}^{2}-k_{y}^{2}\right)+2 j \gamma_{3} k_{x} k_{y}\right]
\end{gathered}
$$

in which $m_{0}$ is the mass of a free electron, $\gamma_{1}, \gamma_{2}, \gamma_{3}$ are the Luttinger parameters and $a_{v}, b$ are the deformation potentials [34] with different values in QWs and barriers, and the lattice mismatch strain

$$
\epsilon_{x x}=\epsilon_{y y}=\frac{a_{0}-a}{a}, \quad \epsilon_{y y}=-\frac{2 C_{12}}{C_{11}} \epsilon_{x x}
$$

with $a_{0}, a$ being the lattice constants of the substrate (or buffer) and the layer material, and $C_{11}$ and $C_{12}$ the stiffness constants. 
The Hamiltonian in Eq.(9) operates on wavefunctions that are combinations of six mutually orthogonal $\mathrm{HH}\left(\mid \frac{3}{2}, \pm \frac{3}{2}>\right)$, LH $\left(\mid \frac{3}{2}, \pm \frac{1}{2}>\right)$, and SO $\left(\mid \frac{1}{2}, \pm \frac{1}{2}>\right)$ Bloch functions

$$
\begin{gathered}
\Psi_{i}(\boldsymbol{r}, z)=e^{j \boldsymbol{k} \cdot \boldsymbol{r}}\left[\chi_{1}(z)\left|\frac{3}{2}, \frac{3}{2}>+\chi_{2}(z)\right| \frac{3}{2}, \frac{1}{2}>+\chi_{3}(z)\left|\frac{3}{2},-\frac{1}{2}>+\chi_{4}(z)\right| \frac{3}{2},-\frac{3}{2}>\right. \\
\left.+\chi_{5}(z)\left|\frac{1}{2},-\frac{1}{2}>+\chi_{6}(z)\right| \frac{1}{2},-\frac{1}{2}>\right]
\end{gathered}
$$

where $\chi_{n}(z), n=1,2, \cdots, 6$ forms a six-component envelope-function vector $\chi(z)$. Each component in a given region $l$ of the QW structure (either QW or barrier), $d_{l}<z<d_{l+1}$, is a superposition of the forward and backward propagations identical to Eq.(4) with constants $A_{n, l}$ and $B_{n, l}, n=1,2, \cdots, 6$ that can be fixed by the continuity equations that require at each interface $z=d_{l}$,

$$
\chi(z) \text { and }\left[\begin{array}{cccccc}
p+q & -s & 0 & 0 & -\frac{1}{\sqrt{2}} s & 0 \\
-s^{\dagger} & p-q & 0 & 0 & -\sqrt{2} q & \sqrt{\frac{3}{2} s} \\
0 & 0 & p-q & s & \sqrt{\frac{3}{2}} s^{\dagger} & \sqrt{2} q \\
0 & 0 & s^{\dagger} & p+q & 0 & -\frac{1}{\sqrt{2}} s^{\dagger} \\
-\frac{1}{\sqrt{2}} s^{\dagger} & -\sqrt{2} q & \sqrt{\frac{3}{2}} s & 0 & p & 0 \\
0 & \sqrt{\frac{3}{2}} s^{\dagger} & \sqrt{2} q & -\frac{1}{\sqrt{2}} s & 0 & p
\end{array}\right] \chi(z) \text { continuous }
$$

where

$$
\begin{gathered}
p=\gamma_{1} \frac{\partial}{\partial z} \\
q=-2 \gamma_{2} \frac{\partial}{\partial z} \\
s=-\sqrt{3} j \gamma_{3}\left(k_{x}-j k_{y}\right)
\end{gathered}
$$

to maintain undisruptive carrier distribution and current across the interface.

It is important to point out that when the above described algorithms are used for the situation where an electric field is applied along the growth direction $z$, it is necessary to digitize the potential term $V_{c}(z)$ and $V_{v}(z)$, i.e. the regions that are used in Eq.(4) are no longer defined by the QW and barrier boundaries; instead, there could be many regions within each QW or barrier depending on the number of digitization steps used to satisfy the accuracy requirement.

This procedure applied at each wave vector point $\left(\boldsymbol{k}=k_{x} \hat{x}+k_{y} \hat{y}\right)$ produces the in-plane dispersion for each subband. An example is illustrated in Fig. 5 for a $70 \AA / 50 \AA$ 
$\mathrm{GaAs} / \mathrm{Al}_{0.3} \mathrm{Ga}{ }_{0.7} \mathrm{As} \mathrm{SL}$ [35]. In-plane dispersions of three subbands (two for $\mathrm{HH}$ and one for $\mathrm{LH}$ ) are shown where strong nonparabolicity is demonstrated. It can be seen from Fig. 5 that the band nonparabolicity could be so severe that the LH subband maximum is no longer at the $\Gamma$-point which leads to useful valence QCL design applications in Section IV.

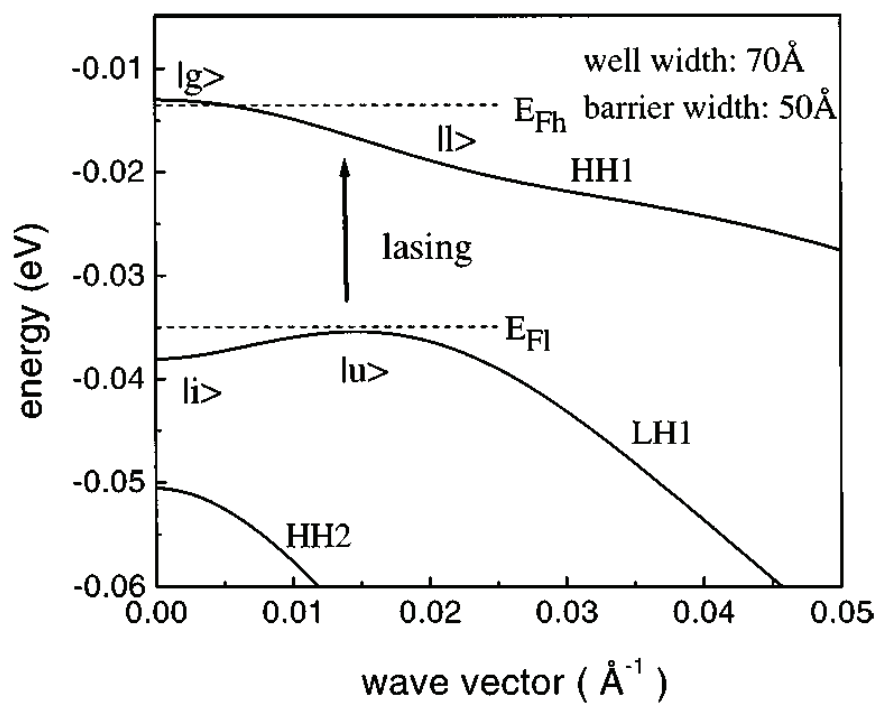

Fig. 5. In-plane dispersions of subbands HH1, LH1, and HH2 for a $70 \AA$ / $50 \AA$ GaAs/AlGaAs SL [35].

\subsection{Optical gain}

For lasing to occur between two subbands, it is necessary to induce stimulated emission between them. To sustain such emission of photons, there must be sufficient optical gain to compensate various losses in the laser structure. The intersubband optical gain can be obtained by analyzing transition rates between two subbands.

According to the Fermi Golden rule, the transition rate between two discrete states 1 and 2 that are coupled by a perturbation electro-magnetic (EM) field with a frequency of $\omega$ is

$$
g_{12}=\frac{2 \pi}{\hbar}\left|H_{m}\right|^{2} \delta\left(E_{2}-E_{1}-\hbar \omega\right)
$$

where $H_{m}=\left\langle 1\left|H_{e x}\right| 2\right\rangle$ is the transition matrix element under the influence of a perturbation Hamiltonian $H_{e x}$ between the two states with an exact transition energy $E_{2}-E_{1}$ in the absence of any broadening. In reality, the transition line $E_{2}-E_{1}$ is not infinitely sharp and is always broadened. As a result, $E_{2}-E_{1}$ is not known exactly, instead a probability for it to appear in the energy interval $E \rightarrow E+d E$ is described. In the case of homogeneous broadening, this probability should be given as $L(E) d E$ with the Lorentzian lineshape centered at some peak transition energy $E_{0}$

$$
L(E)=\frac{\Gamma / 2 \pi}{\left(E-E_{0}\right)^{2}+\Gamma^{2} / 4}
$$


where $\Gamma$ is the full width at half maximum (FWHM) that characterizes the broadening due to various homogeneous processes that include collisions and transitions. The transition rate in Eq.(15) should thus be modified by an integral that takes into account of this broadening as

$$
g_{12}=\frac{2 \pi}{\hbar} \int\left|H_{m}\right|^{2} \delta(E-\hbar \omega) L(E) d=\frac{2 \pi}{\hbar}\left|H_{m}\right|^{2} \frac{\Gamma / 2 \pi}{\left(\hbar \omega-E_{0}\right)^{2}+\Gamma^{2} / 4}
$$

essentially replacing the $\delta$-function in Eq.(15) with the Lorentzian lineshape Eq.(16).

In the presence of an EM field with an optical potential vector $\boldsymbol{A}$ in a medium with isotropic effective mass, the perturbation Hamiltonian $H_{e x}$ that describe the interaction between the field and electron in isotropic subbands is

$$
H_{e x}=\frac{e \boldsymbol{A} \cdot \boldsymbol{P}}{m_{e}^{*}}
$$

where $\boldsymbol{P}$ is the momentum operator.

From Eq.(18), it is not difficult to see that the selection rules for intersubband transitions in the conduction band are such that only those EM fields that are polarized in the growth direction $(z)$ can induce optical transitions. The transition matrix element can then be given as

$$
H_{m}=\frac{e A}{m_{e}^{*}} P_{12}
$$

where the momentum matrix element

$$
P_{12}=\left\langle\varphi_{1}\left|-j \hbar \frac{\partial}{\partial z}\right| \varphi_{2}\right\rangle
$$

is evaluated as the envelope function overlap between the two subbands, which is related to the dipole matrix element [36]

$$
z_{12}=\left\langle\varphi_{1}|z| \varphi_{2}\right\rangle=\frac{i \hbar}{m_{0} E_{12}} P_{12}
$$

and to the oscillator strength [37]

$$
f_{12}=\frac{2 m_{0}}{m_{e}^{* 2} E_{12}}\left|P_{12}\right|^{2} .
$$

It is not difficult to see from Eq.(17) that the transition rate induced by an EM field between two eigen states is the same for upward and downward transitions. Now let us apply Eq.(17) to intersubband transitions between the upper subband 2 and lower subband 1 in the conduction band (Fig. 3). Since momentums associated with photons are negligible, all photon-induced transitions are vertical in $\boldsymbol{k}$-space. It is therefore possible to obtain a net downward transition rate (in the units of number of transitions per unit time per unit sample area) between the two subbands by evaluating the following integral

$$
g_{\text {net }}=\int g_{12}\left\{f_{2}\left(E_{2, \boldsymbol{k}}\right)\left[1-f_{1}\left(E_{1, \boldsymbol{k}}\right)\right]-f_{1}\left(E_{1, \boldsymbol{k}}\right)\left[1-f_{2}\left(E_{2, \boldsymbol{k}}\right)\right]\right\} \rho_{r}\left(E_{2, \boldsymbol{k}}-E_{1, \boldsymbol{k}}\right) d\left(E_{2, \boldsymbol{k}}-E_{1, \boldsymbol{k}}\right)
$$


where $f_{1}\left(E_{1, k}\right)$ and $f_{2}\left(E_{2, \boldsymbol{k}}\right)$ are the electron occupation probabilities of those states at the same $\boldsymbol{k}$ in subbands 1 and 2, respectively, and $\rho_{r}\left(E_{2, \boldsymbol{k}}-E_{1, k}\right)$ is the reduced density of states (DOS) between $E_{1, \boldsymbol{k}}$ and $E_{2, \boldsymbol{k}}$, which is equal to DOS of subbands $1\left(\rho_{1}\right)$ and $2\left(\rho_{2}\right)$ when they are parallel, $\rho_{r}=\rho_{1}=\rho_{2}=m_{e}^{*} / \pi \hbar^{2}$. Since the Lorentzian lineshape in Eq.(23) should be much broader than the spread of the energy transitions between the two parallel subbands which can be approximated as sharply centered at the subband separation at their energy minima $E_{12}=E_{2}-E_{1}$. Thus,

$$
\begin{gathered}
g_{\text {net }}=\frac{2 \pi}{\hbar}\left|H_{m}\right|^{2} \frac{\Gamma / 2 \pi}{\left(\hbar \omega-E_{12}\right)^{2}+\Gamma^{2} / 4}\left[\int f_{2}\left(E_{2, \boldsymbol{k}}\right) \rho_{2} d E_{2, \boldsymbol{k}}-\int f_{1}\left(E_{1, \boldsymbol{k}}\right) \rho_{1} d E_{1, \boldsymbol{k}}\right] \\
=\frac{2 \pi}{\hbar}\left|H_{m}\right|^{2} \frac{\Gamma / 2 \pi}{\left(\hbar \omega-E_{12}\right)^{2}+\Gamma^{2} / 4}\left(N_{2}-N_{1}\right)
\end{gathered}
$$

where $N_{1}$ and $N_{2}$ are the total electron densities in subband 1 and 2 per unit area, respectively.

The optical gain coefficient $\gamma$ that describes the increase of the EM field intensity, $I$, as $\gamma=I^{-1} d I / d z$ can be defined as power increase per unit volume divided by the intensity, which in turn can be expressed in terms of the net downward transition rate Eq.(24) using the momentum and dipole matrix element relation

$$
\gamma(\omega)=\frac{g_{\text {net }} \hbar \omega}{I L_{p}}
$$

where $L_{p}$ is the length of the QW structure that is equal to the length of one period in case of QCLs. In order to relate the EM field intensity $I$ that propagates in in-plane with the optical potential $\mathbb{A}$ polarized along $z$, a real expression for the potential $\mathbb{A}$ has to be used

$$
\mathbb{A}=A_{0} \cos (\boldsymbol{\beta} \cdot \boldsymbol{r}-\omega t) \hat{\mathbf{z}}=\frac{1}{2} A_{0} \hat{\mathbf{z}}\left[e^{j(\boldsymbol{\beta} \cdot \boldsymbol{r}-\omega t)}+e^{-j(\boldsymbol{\beta} \cdot \boldsymbol{r}-\omega t)}\right]
$$

where $\boldsymbol{\beta}$ is the in-plane propagation wave vector of the EM field. It is easy to see that only one of the two terms on the right side of Eq.(26) couples with subbands 1 and 2, $E_{2}-E_{1}=$ $\hbar \omega$. Thus, the optical potential that participates in the transition matrix Eq.(19) is only half of its real amplitude, $A=A_{0} / 2$. Since the EM field intensity $I$ is related to the optical potential amplitude $A_{0}$ as $I=\varepsilon_{0} c n_{e f f} A_{0}^{2} \omega^{2} / 2$, Eq.(25) can be written as

$$
\begin{aligned}
\gamma(\omega) & =\frac{e^{2}\left|P_{12}\right|^{2}}{2 \varepsilon_{0} c n_{e f f} m_{e}^{* 2} \omega L_{p}} \frac{\Gamma}{\left(\hbar \omega-E_{12}\right)^{2}+\Gamma^{2} / 4}\left(N_{2}-N_{1}\right) \\
& =\frac{e^{2} m_{0}^{2} \omega z_{12}^{2}}{2 \varepsilon_{0} c n_{e f f} m_{e}^{* 2} L_{p}} \frac{\Gamma}{\left(\hbar \omega-E_{12}\right)^{2}+\Gamma^{2} / 4}\left(N_{2}-N_{1}\right)
\end{aligned}
$$

where $c$ is the speed of light in free space and $n_{e f f}$ is the effective index of refraction of the QCL dielectric medium. The population inversion $N_{2}-N_{1}>0$ is clearly necessary in order to achieve positive gain which peaks at the frequency $\omega_{0}=E_{12} / \hbar$ with a value of

$$
\gamma\left(\omega_{0}\right)=\frac{2 e^{2} m_{0}^{2} \omega z_{12}^{2}}{\varepsilon_{0} c n_{e f f} m_{e}^{* 2} \Gamma L_{p}}\left(N_{2}-N_{1}\right)
$$

For transitions between valence subbands with nonparallel dispersions and strong mixing between $\mathrm{HH}, \mathrm{LH}$, and $\mathrm{SO}$ bands, we have to re-examine the intersubband transition rate. 
Consider the intersubband transition in Fig. 5 from the upper state $\mid u>$ in subband LH1 to the lower state $\mid l>$ in subband HH1, if the spread of intersubband transitions is wide enough compared to the homogeneous broadening, the Lorentzian lineshape in the net downward transition rate Eq.(23) can be approximated as a $\delta$-function yielding

$$
g_{\text {net }}^{(v)}=\left.\frac{2 \pi}{\hbar}\left|H_{m}^{(v)}\right|^{2} \rho_{r}\left(E_{l}-E_{h}\right)\left[f_{L H}\left(E_{l}\right)-f_{H H}\left(E_{h}\right)\right]\right|_{E_{l}-E_{h}=\hbar \omega}
$$

where $\rho_{r}\left(E_{l}-E_{h}\right)$ is the reduced DOS for the transition between subbands LH1 and HH1, $f_{L H}\left(E_{l}\right)$ and $f_{H H}\left(E_{h}\right)$ are hole occupation probabilities at states with energy $E_{l}$ and $E_{h}$ in subband LH1 and HH1, respectively, at the same in-plane wave vector $\boldsymbol{k}$ separated by a photon energy $\hbar \omega$, and the optical transition matrix between LH1 and HH1 taking into account of the mixing

$$
H_{m}^{(v)}=\sum_{n=1}^{6} \frac{e A}{m_{n}^{*}}\left\langle\chi_{n}^{(l)}\left|-j \hbar \frac{\partial}{\partial z}\right| \chi_{n}^{(h)}\right\rangle
$$

where $\chi_{n}^{(l)}$ and $\chi_{n}^{(h)}$ are respectively the $n$-th component of the envelope function vectors for subband LH1 and HH1 as defined in Eq.(12), and $m_{n}^{*}$ are the corresponding hole effective mass in $z$-direction with $m_{1,4}^{*}=m_{0} /\left(\gamma_{1}-2 \gamma_{2}\right)$ for $\mathrm{HH}, m_{2,3}^{*}=m_{0} /\left(\gamma_{1}+2 \gamma_{2}\right)$ for LH, and $m_{5,6}^{*}=m_{0} / \gamma_{1}$ for SO. The optical gain can then be expressed

$$
\begin{aligned}
\gamma^{(v)} & =\left.\frac{\pi e^{2}}{\varepsilon_{0} c n_{e f f} \omega L_{p}}\left|\sum_{n=1}^{6} \frac{P_{n}^{(l h)}}{m_{n}^{*}}\right|^{2} \rho_{r}\left(E_{l}-E_{h}\right)\left[f_{L H}\left(E_{l}\right)-f_{H H}\left(E_{h}\right)\right]\right|_{E_{l}-E_{h}=\hbar \omega} \\
& =\left.\frac{\pi e^{2} m_{0}^{2} \omega}{\varepsilon_{0} c n_{e f f} L_{p}}\left|\sum_{n=1}^{6} \frac{z_{n}^{(l h)}}{m_{n}^{*}}\right|^{2} \rho_{r}\left(E_{l}-E_{h}\right)\left[f_{L H}\left(E_{l}\right)-f_{H H}\left(E_{h}\right)\right]\right|_{E_{l}-E_{h}=\hbar \omega}
\end{aligned}
$$

in terms of momentum matrix $P_{n}^{(l h)}=\left\langle\chi_{n}^{(l)}\left|-j \hbar \frac{\partial}{\partial z}\right| \chi_{n}^{(h)}\right\rangle$ as well as dipole matrix elements $z_{n}^{(l h)}=\left\langle\chi_{n}^{(l)}|z| \chi_{n}^{(h)}\right\rangle$ between the same $n$-th component of the envelope function vectors of the two valence subbands.

In comparison with the optical gain Eq.(28) for the conduction subbands, we can see that it is not necessary to have total population inversion, $N_{l}-N_{h}>0$, in order to have positive gain between the valence subbands. Instead, all we need is local population inversion $\left.\left[f_{L H}\left(E_{l}\right)-f_{H H}\left(E_{h}\right)\right]\right|_{E_{l}-E_{h}=\hbar \omega}>0$ in the region where the intersubband transition takes place (those states near $\mid u>$ and $\mid l>$ in Fig. 5).

\subsection{Intersubband lifetimes}

It has been established in Eqs.(27) and (28) that the population inversion between the upper (2) and lower (1) subbands, $N_{2}-N_{1}>0$, is necessary in order to obtain optical gain. But what determines the population inversion? This question is answered with the analysis of lifetimes of these subbands as a result of various intersubband relaxation mechanisms including carrier-phonon, carrier-carrier, impurity, and interface roughness scattering processes. Among them, phonon scattering is the dominant process, especially when the energy separation between the two subbands exceeds that of an optical phonon, in which 
case the transitions from upper to lower subband are highly efficient with the emission of optical phonons. Different from the optical transitions, these scattering processes do not necessarily occur as vertical transitions in $\boldsymbol{k}$-space. In the case of phonon scattering, the conservation of in-plane momentum can be satisfied by a wide range of momentum of involved phonons as shown in Fig. 6(a) where intersubband as well as intrasubband transitions due to phonon scattering are illustrated.

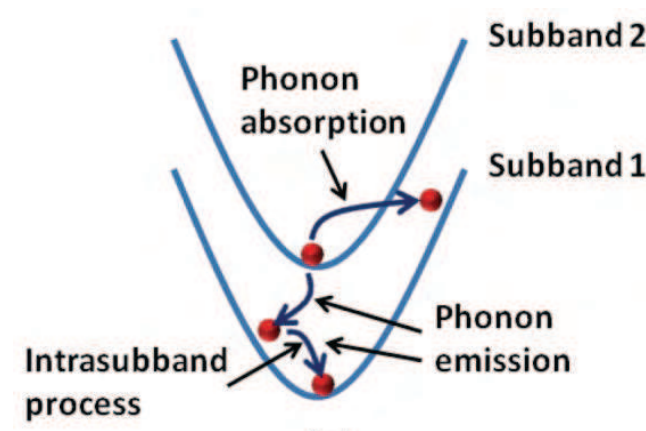

(a)

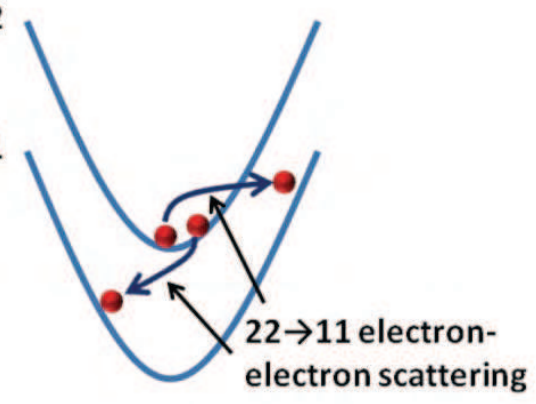

(b)

Fig. 6. (a) Intersubband and intrasubband transitions due to electron-phonon scattering (b) the $22 \rightarrow 11$ transition induced by the electron-electron scattering.

Up to now, practically all approaches in developing Si-based QCLs are based on materials from group-IV, mostly Si, Ge, SiGe alloy, and more recently, SiGeSn alloy. Different from the polar III-V and II-VI semiconductors, group-IV materials are nonpolar. The carrier scatterings by nonpolar optical phonons are much slower than those due to polar optical phonons [38]. Starting from Fermi Gold rule Eq.(15), the scattering rate for a carrier in subband 2 with the in-plane wave vector $\boldsymbol{k}$ to subband 1 with $\boldsymbol{k}^{\prime}$ by a phonon with an energy $\hbar \omega_{\boldsymbol{Q}}$ and wave vector, $\boldsymbol{Q}=\boldsymbol{q}+q_{z} \hat{\mathbf{z}}$, can be expressed as an integral over all the participating phonon states

$$
\frac{1}{\tau_{12}}=\frac{2 \pi}{\hbar} \int\left|H_{e p}\right|^{2} \delta\left(E_{2, \boldsymbol{k}}-E_{1, \boldsymbol{k}^{\prime}} \mp \hbar \omega_{Q}\right) d N_{f}
$$

where $H_{e p}$ is the electron-phonon interaction matrix element, the carrier energies $E_{1, \boldsymbol{k}}$, and $E_{2, \boldsymbol{k}}$ are given by Eq.(1) for conduction subbands, but for valence subbands, they need to be obtained by the $\boldsymbol{k} \cdot \boldsymbol{p}$ method described above. We will proceed with the following approximations: 1) all phonons are treated to be bulk-like by neglecting the phonon confinement effect in QW structures, 2) energies of acoustic phonons are negligible $\hbar \omega_{Q} \approx 0$, and 3) optical phonon energies are taken as a constant $\hbar \omega_{Q} \approx \hbar \omega_{0}$. The matrix element of carrier-phonon interaction for different type of phonons can be written as $[39,40]$

$$
\left|H_{e p}\right|^{2}=\left\{\begin{array}{lr}
\frac{\Xi^{2} K_{B} T}{2 c_{L} \Omega} \delta_{\boldsymbol{q}, \pm\left(\boldsymbol{k}^{\prime}-\boldsymbol{k}\right)}\left|G_{12}\left(q_{z}\right)\right|^{2}, & \text { acoustic phonon } \\
\frac{\hbar D^{2}}{2 \rho \omega_{0} \Omega} \delta_{\boldsymbol{q}, \pm\left(\boldsymbol{k}^{\prime}-\boldsymbol{k}\right)}\left[n\left(\omega_{0}\right)+\frac{1}{2} \mp \frac{1}{2}\right]\left|G_{12}\left(q_{z}\right)\right|^{2}, & \text { nonpolar optical phonon }
\end{array}\right.
$$


where the upper sign is for absorption and lower for emission of one phonon, $K_{B}$ is the Boltzmann constant, $\Omega$ is the volume of the lattice mode cavity, $c_{L}$ is the elastic constant for acoustic mode, $\Xi$ and $D$ are the acoustic and optical deformation potential, respectively, and $n\left(\omega_{0}\right)$ is the number of optical phonons at temperature $T$,

$$
n\left(\omega_{0}\right)=\frac{1}{\exp \left(\hbar \omega_{0} / K_{B} T\right)-1} .
$$

The wavefunction interference effect between conduction subbands is

$$
G_{12}\left(q_{z}\right)=\left\langle\varphi_{1}\left|e^{j q_{z} z}\right| \varphi_{2}\right\rangle
$$

and between valence subbands is

$$
G_{12}\left(q_{z}\right)=\sum_{n=1}^{6}\left\langle\chi_{n}^{(1)}\left|e^{j q_{z} z}\right| \chi_{n}^{(2)}\right\rangle .
$$

The Kronecker symbol $\delta_{q, \pm\left(\boldsymbol{k}^{\prime}-\boldsymbol{k}\right)}$ in the matrix element Eq.(33) represents the in-plane momentum conservation $\boldsymbol{k}^{\prime}=\boldsymbol{k} \pm \boldsymbol{q}$.

Since phonon modes have density of states $\Omega /(2 \pi)^{3}$, the participating phonon states in the integral Eq.(32) can be expressed as

$$
d N_{f}=\frac{\Omega}{(2 \pi)^{3}} q d q d \theta d q_{z}
$$

where $\theta$ is the angle between $\boldsymbol{k}$ and $\boldsymbol{q}$. For conduction subbands with a parabolic dispersion Eq.(1), the phonon scattering rate Eq.(32) can be evaluated analytically

$$
\frac{1}{\tau_{12}}=\left\{\begin{array}{lr}
\frac{\Xi^{2} K_{B} T m_{e}^{*}}{4 \pi c_{L} \hbar^{3}} \int\left|G_{12}\left(q_{z}\right)\right|^{2} d q_{z}, & \text { acoustic } \\
\frac{D^{2} m_{e}^{*}\left[n\left(\omega_{0}\right)+\frac{1}{2} \mp \frac{1}{2}\right]}{4 \pi \rho \hbar^{2} \omega_{0}} \int\left|G_{12}\left(q_{z}\right)\right|^{2} d q_{z}, & \text { nonpolar optical. }
\end{array}\right.
$$

But for valence subbands where there is a strong nonparabolicity, Eq.(32) can no longer be integrated analytically. However, if we take the wave vector of the initial state in subband 2 to be at the $\Gamma$-point $\boldsymbol{k}=0$, then the phonon wave vector $\boldsymbol{q}=\boldsymbol{k}^{\prime}$, Eq.(38) can still be used to evaluate the phonon scattering rate between valence subbands by substituting the effective mass with some average effective mass in the final subband 1 .

The phonon scattering rate in Eq.(38) has been used to compare the lifetimes of two similar three-level systems, SiGe/Si and GaAs/AlGaAs, as shown in Fig. 7(a) [38]. The lifetime difference between the upper (3) and lower (2) subband is calculated as the function of the transition energy $E_{3}-E_{2}$ which is varied by changing the barrier width between the two QWs that host the two subbands. The main result in Fig. 7(b) is that the lifetimes in the SiGe system can be an order of magnitude longer than in the GaAs/AlGaAs system because of SiGe's lack of polar optical phonons. This property can potentially lead to significantly reduced lasing threshold for the SiGe system. The sudden drops in the lifetimes have to do with the shifting of subband energy separations $E_{2}-E_{1}$ and $E_{3}-E_{2}$, to either below or above the optical phonon energy. 


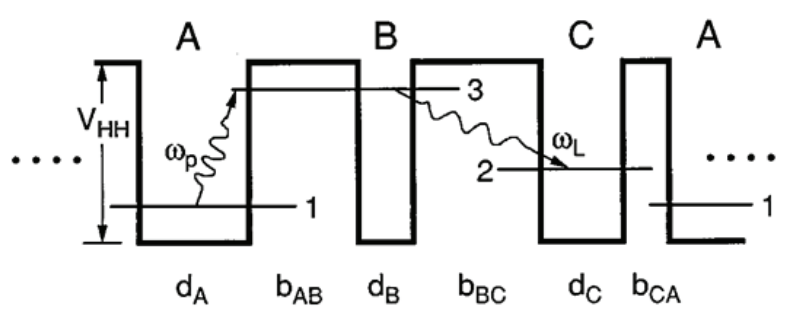

(a)

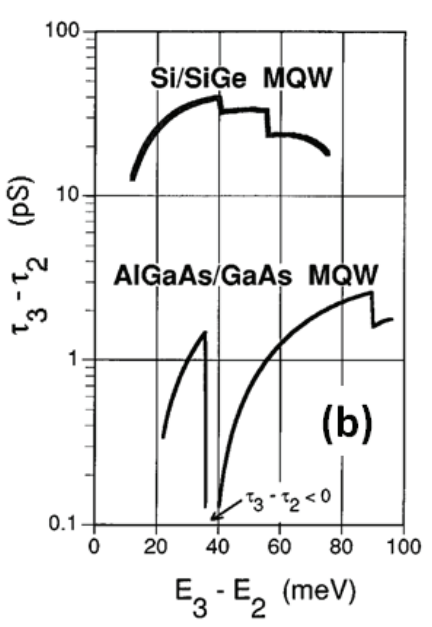

Fig. 7. (a) HH valence band diagram of one period of a SiGe/Si SL with hole energy increase in the upward direction, and (b) comparison of Lifetime difference $\left(\tau_{3}-\tau_{2}\right)$ between the $\mathrm{SiGe} / \mathrm{Si}$ and GaAs/AlGaAs SL (in a similar three scheme) as a function of the transition energy $\left(E_{3}-E_{2}\right)[38]$.

Among the different phonon scattering processes - emission and absorption of acoustic and optical phonons, the emission of an optical phonon is by far the fastest process. But in far-IR QCLs when the subband energy separation is less than the optical phonon energy and the emission of an optical phonon is forbidden, phonon scattering may no longer be the dominant relaxation mechanism. Other scattering mechanisms need to be taken into consideration, such as the carrier-carrier [41], impurity [42], and interface roughness scatterings [43], all of which are elastic processes. The carrier-carrier scattering is a twocarrier process that is particularly important when carrier concentration is high which increases the possibility of two carriers interacting with each other. There are many possible outcomes as a result of this interaction in inducing intersubband as well as intrasubband transitions. Among them, the $22 \rightarrow 11$ process where both carriers originally in subband 2 end up in subband 1 is the most efficient one in terms of inducing intersubband transitions (Fig. 6(b)). It has been reported in experiment that the intersubband transition times on the order of tens of ps have been observed for carrier densities of $10^{9} \sim 10^{11} / \mathrm{cm}^{2}$ in GaAs/AlGaAs QWs [44]. In QCLs, since doping is mostly introduced away from the active region where optical transitions take place, impurity scattering does not seem to play a major role in determining the lifetimes of laser subbands, however, its influence on carrier transport in the injection region can be rather important. Interface roughness depends strongly on the process of structural growth, its impact on scattering should be more significant on narrow QWs, particularly for those transitions between two wavefunctions that are localized in MQWs that span across several interfaces.

\section{Valence band SiGe QCLs}

Up to now, all of the demonstrated QCLs are based on epitaxially grown III-V semiconductor heterostructures such as GaInAs/AlInAs, GaAs/AlGaAs, and InAs/AlSb, 
using electron subbands in conduction band. With the promise of circumventing the indirectness of $\mathrm{Si}$ band gap, a SiGe/Si laser based on intersubband transitions was first proposed by Sun et al in 1995 [38] where a comparative study was performed between the $\mathrm{SiGe/Si}$ and GaAs/AlGaAs systems. Since then there has been a series of theoretical and experimental investigations aimed at producing Si-based QCLs. A natural choice of the material system is SiGe because $\mathrm{Si}$ and $\mathrm{Ge}$ are both group-IV elements, SiGe alloys have been routinely deposited on $\mathrm{Si}$ to produce heterojunction bipolar transistors or as a straininducing layer for CMOS transistors [45].

While QCLs based on SiGe alloys can be monolithically integrated on Si if successfully developed, there are significant challenges associated with this material system. First, there is a $4 \%$ lattice mismatch between $\mathrm{Si}$ and Ge. Layers of $\mathrm{Si}_{1-x} \mathrm{Ge}_{\mathrm{x}}$ alloys deposited on $\mathrm{Si}$ substrates induce strain which can be rather significant in QCLs because a working structure typically consists of at least hundreds of layers with a total thickness that easily exceeds the critical thickness above which the built-in strain simply relaxes to develop defects in the structure. In dealing with the issue of strain in SiGe/Si QC structures, one popular approach is to use strain balanced growth where the compressively strained $\mathrm{Si}_{1-x} \mathrm{Ge}_{\mathrm{x}}$ and tensile strained $\mathrm{Si}$ are alternately stacked on a relaxed $\mathrm{Si}_{1-\mathrm{y}} \mathrm{Ge}_{\mathrm{y}}$ buffer deposited on a $\mathrm{Si}$ substrate where the buffer composition $(y<x)$ is chosen to produce strains in $\mathrm{Si}_{1-\mathrm{x}} \mathrm{Ge}_{\mathrm{x}}$ and $\mathrm{Si}$ that compensate each other, so that the entire structure maintains a neutral strain profile $[46,47]$. Strain balanced structures have effectively eliminated the limitations of critical thickness and produced high quality SiGe/Si structures consisting of nearly 5000 layers (15 $\mu \mathrm{m})$ by chemical vapor deposition [48].

Second, the band offsets between compressively strained SiGe and tensile strained Si or between SiGe of different alloy compositions is such that the conduction band QWs are shallow, and nearly all band offsets are in valence band. Practically all of the investigations of SiGe QCLs are focused on intersubband transitions in the valence band. But the valence subband structure is much more complex in comparison with the conduction subbands because of the mixing between the $\mathrm{HH}, \mathrm{LH}$ and SO bands. Their associated subbands are closely intertwined in energy making the design of valence QCLs extremely challenging. Third, any valence QCL design in general has to inevitably involve $\mathrm{HH}$ subbands since they occupy lower energies relative to LH subbands because of their large effective mass. In SiGe, the HH effective mass is high $\left(\sim 0.2 m_{e}\right)$, which leads to small IST oscillator strength between the laser states and poor carrier transport behavior associated with their low mobilities.

The challenge presented by the valence-band mixing also creates an opportunity to engineer desirable subband dispersions such that total population inversion between the subbands becomes unnecessary in a way analogous to the situation in conventional band-to-band lasers discussed in section II. It was reported in QCLs that the population inversion was only established locally in $\boldsymbol{k}$-space in the large $\boldsymbol{k}$-vector region of the conduction subbands because the interactions between the subbands produced nonparallel in-plane dispersions [49]. In comparison with the conduction-band nonparabolicity, this effect is known to be much stronger in the valence band [31]. As a matter of fact, in the valence band of most diamond and zinc-blende semiconductors, $\mathrm{LH}$ and $\mathrm{HH}$ subbands usually anti-cross, and near the point of anti-crossing, the LH subband in-plane dispersion becomes electron-like. Thus, an earlier design was accomplished to effectively tailor the dispersions of two valence subbands in a GaAs/AlGaAs QW (Fig. 5) similar to those of the conduction and valence bands, in which one of the subbands is electron-like and the other hole-like, i.e., one of the 
subbands shall have its effective mass inverted [35]. If we now designate states near the $\Gamma$ point of subband LH1 as the intermediate states, $|i\rangle$, states near the valley (invertedeffective-mass region) of subband LH1 as the upper laser states $|u\rangle$, states in subband HH1 vertically below the valley of subband LH1 as the lower laser states $|l\rangle$, and states near the $\Gamma$-point of subband $\mathrm{HH} 1$ as the ground states $|g\rangle$ (counting the hole energy downward in Fig. 5), we can see that the situation closely resembles the one in the conventional band-toband semiconductor laser. The upper and lower laser states can now be populated and depopulated through fast intrasubband processes, while the lifetime of the upper laser states is determined by a much slower intersubband process between subbands LH1 and $\mathrm{HH} 1$. Such a large lifetime difference between the upper and lower laser states is certainly favorable for achieving population inversion between them.

The inverted mass approach was later on applied to the SiGe system [50,51]. Two slightly different schemes were developed, one utilized the inverted LH effective mass [50], and the other inverted $\mathrm{HH}$ mass [51]. In both cases, the effective mass inversion is the result of strong interaction between the valence subbands. The inverted-effective-mass feature requires the coupled subbands to be closely spaced in energy, typically less than all the optical phonon energies in the SiGe material system ( $37 \mathrm{meV}$ for the Ge-Ge mode, $64 \mathrm{meV}$ for the Si-Si mode, and $51 \mathrm{meV}$ for the Si-Ge mode [52]), which suppresses the nonradiative intersubband transitions due to the optical phonon scattering, but also limits the optical transitions in the $\mathrm{THz}$ regime. The structures under investigation were strain balanced with compressively strained $\mathrm{Si}_{1-\mathrm{x}} \mathrm{Ge}_{\mathrm{x}} \mathrm{QW}$ layers and the tensile strained $\mathrm{Si}$ barrier layers deposited on a relaxed $\mathrm{Si}_{1-y} \mathrm{Ge}_{\mathrm{y}}$ buffer layer $(0<y<x)$ on $\mathrm{Si}$. The in-plane dispersions of the inverted LH scheme are shown in Fig. 8 for a $90 \AA / 50 \AA \mathrm{Si}_{0.7} \mathrm{Ge}_{0.3} / \mathrm{Si}$ super lattice (SL). Three lowest subbands are shown. The numbers 1,2, 3, and 4 indicate how this inverted mass

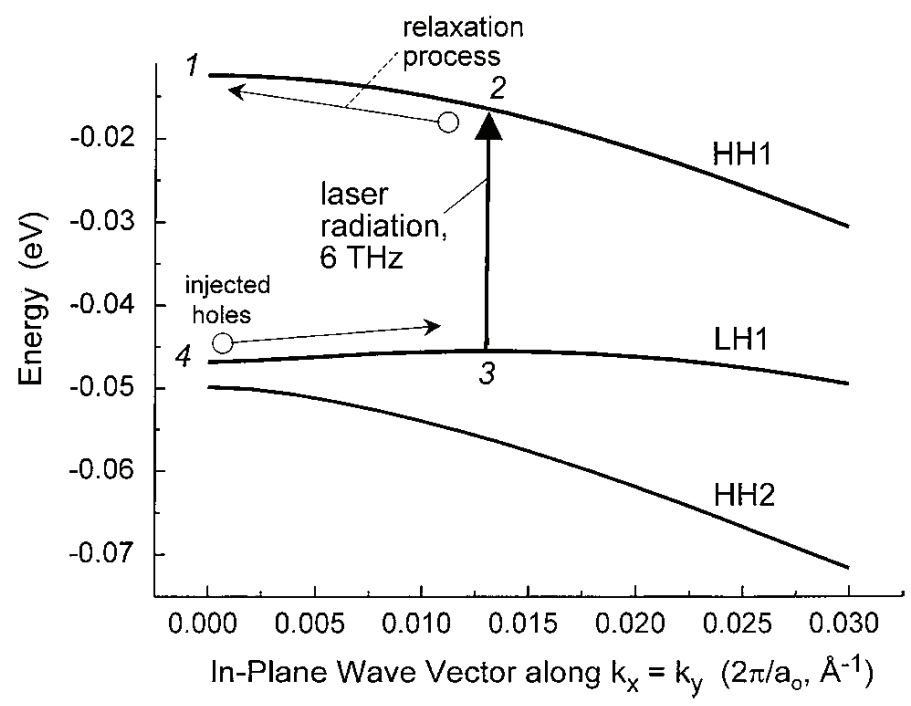

Fig. 8. Dispersions of subbands HH1, LH1 and HH2 in a $90 \AA / 50 \AA \mathrm{Si}_{0.7} \mathrm{Ge}_{0.3} / \mathrm{Si}$ SL strained balanced on a $\mathrm{Si}_{0.81} \mathrm{Ge}_{0.19}$ buffer, obtained with a $6 \times 6$ valence band matrix taking into account $\mathrm{HH}, \mathrm{LH}$ and $\mathrm{SO}$ interactions and strain effect [50]. 
intersubband laser mimics the operation of a conventional band-to-band laser. The lifetime of the upper laser state 3 is long because the intersubband transition energy at $6 \mathrm{THz}$ $(\sim 50 \mu \mathrm{m})$ is below that of optical phonons, allowing only much weaker acoustic phonon scattering between the two subbands. Calculation results have shown that optical gain in excess of $150 / \mathrm{cm}$ can be achieved without total population inversion being established between the $\mathrm{LH}$ and $\mathrm{HH}$ subbands.

The inverted LH effective mass approach utilizes optical transitions between the LH and $\mathrm{HH}$ subband. It can be argued from the component overlap of the envelope functions in Eq.(30) that the optical transition matrix between subbands of different types is always smaller than that between subbands of the same type. We therefore tried to engineer the same inverted effective mass feature between two $\mathrm{HH}$ subbands. The challenge is to lift the LH subband above the HH2 subband. Once again, a strain balanced SL structure is considered but with different SiGe alloy compositions and layer thicknesses [51]. The band structure for a $90 \AA / 35 \AA \mathrm{Si}_{0.8} \mathrm{Ge}_{0.2} / \mathrm{Si}$ SL under an electric bias of $30 \mathrm{kV} / \mathrm{cm}$ is shown in Fig. 9(a) where each QW has two active doublets formed by bringing HH1 and HH2 subbands in the neighboring QWs into resonance under the bias. There is a $3 \mathrm{meV}$ energy split within the doublet. The resulting in-plane dispersions for the two doublets are shown in Fig. 9(b). Simulation results showed that optical gain of $450 / \mathrm{cm}$ at $7.3 \mathrm{THz}$ can be achieved at a pumping current density of $1.5 \mathrm{KA} / \mathrm{cm}^{2}$ at $77 \mathrm{~K}$.
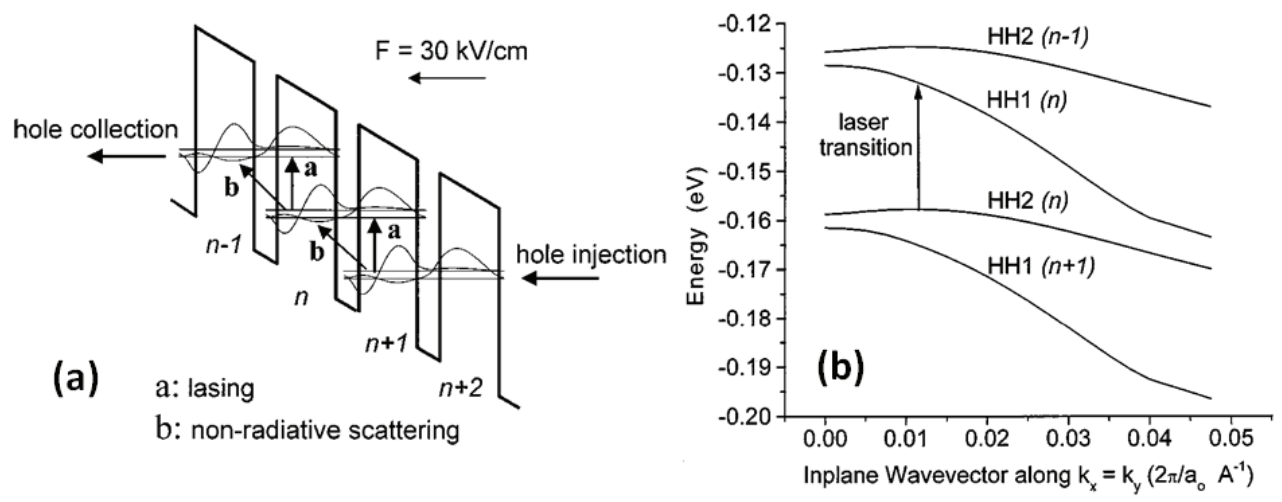

Fig. 9. (a) Band diagram of the $\mathrm{Si}_{0.8} \mathrm{Ge}_{0.2} / \mathrm{Si} \mathrm{SL}$ under an electric bias of $30 \mathrm{kV} / \mathrm{cm}$. The labels $(n-1, n, n+1, \ldots)$ represent the QWs in which the wave functions are localized [51],

(b) Dispersions of the four levels (two doublets) in a QW.

Electroluminescence (EL) from a SiGe/Si quantum cascade emitter was first demonstrated in a $\mathrm{SiGe} / \mathrm{Si}$ quantum cascade emitter using $\mathrm{HH}$ to $\mathrm{HH}$ transitions in the mid-IR range in 2000 [53]. Since then several groups have observed EL from the same material system with different structures. EL emissions have been attributed to various optical transitions including $\mathrm{HH}$-to-HH [54], LH-to-HH [55], and HH-to-LH [56], with emission spectra ranging from mid IR to $\mathrm{THz}(8 \sim 250 \mu \mathrm{m})$. But lasing has not been observed. Improvement on the QCL design has been to be made. One of the most successful III-V QCL designs has been the approach of bound-to-continuum where the lower laser state sitting at the top of a miniband is delocalized over several QWs while the upper laser state is a bound state in the minigap as illustrated in Fig. 10 [57,58]. Electrons that are injected into the bound upper 
state 2 are prevented from escaping the bound state by the minigap, and then undergo lasing transitions to a lower state 1 . The depopulation of the lower state 1 is accelerated through the efficient miniband carrier transport. Such a design has led to improved performance in terms of operating temperature as well as output power for III-V QCLs. A similar bound-to-continuum design has been implemented in SiGe with both bound and continuum formed by $\mathrm{HH}$ states, once again showing just EL with no lasing [59]. It is believed that in this structure LH states are mixed within the HH states. Although the impact of this intermixing has not been fully understood, these LH states can in principle present additional channels for carriers to relax from the upper laser state reducing its lifetime. An improved version has been sought after by lifting the LH states out of all involved $\mathrm{HH}$ states for bound and continuum with the use of strain, as a result, a clear intersubband TM polarized EL is shown suggesting that LH states have been pushed away from the $\mathrm{HH}$ radiative transitions [60].

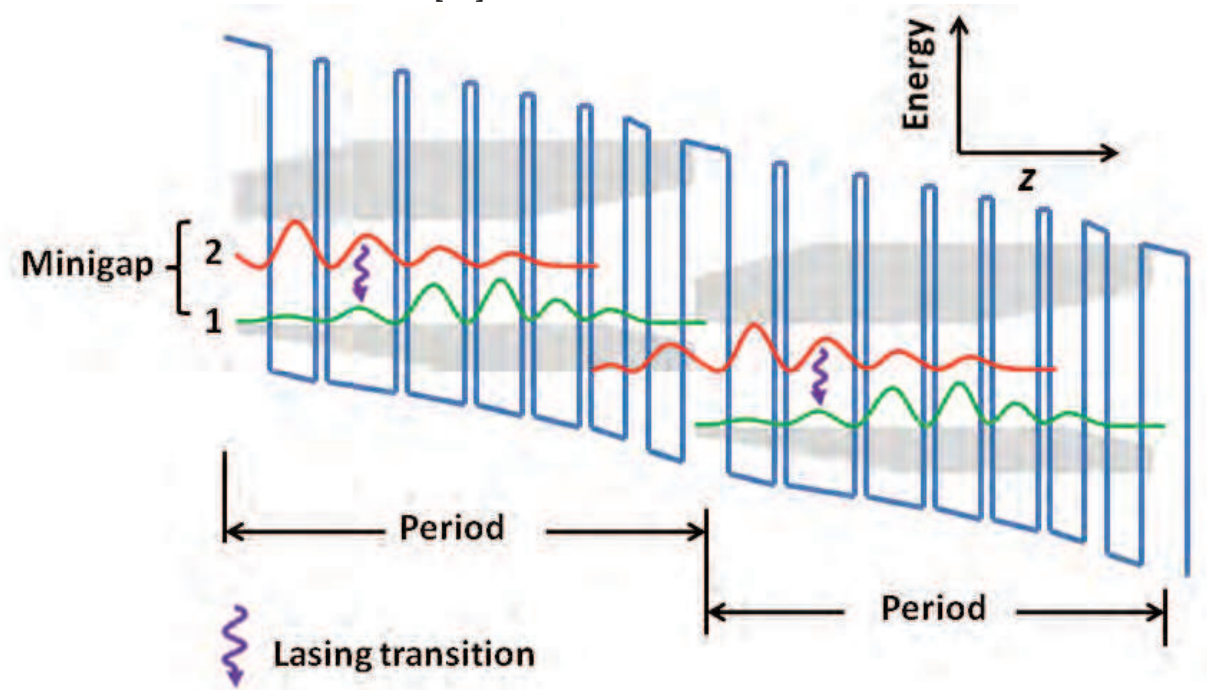

Fig. 10. Illustration of two periods of a bound-to-continuum QCL. Lasing transition occurs between an isolated bound upper state 2 (formed in the minigap) and a delocalized lower state 1 (sitting on top of a miniband).

Nearly a decade has passed since the first experimental demonstration of EL from a SiGe/Si quantum cascade emitter [53]. During this period III-V QCLs have been improved dramatically to allow for commercialization and system integration for various applications, however there are still no SiGe QCLs. The seemingly inherent difficulties with the valence QCL approach have propelled some researchers back into the conduction band to look for solutions.

\section{Conduction band Si-based QCLs}

Before QCLs can be designed using conduction subbands, there must be sufficient conduction band offset. Contrary to the situation in compressively strained $\mathrm{Si}_{1-\mathrm{x}} \mathrm{Ge}_{\mathrm{x}}$, tensile strained $\mathrm{Si}_{1-\mathrm{x}} \mathrm{Ge}_{\mathrm{x}}$ can have larger conduction band offset, but the conduction band minima 
occur at the two $\Delta_{2}$-valleys whose effective mass (longitudinal) along the growth direction is very heavy $\left(m_{l} \sim 0.9 m_{0}\right)$ resulting in small oscillator strength and poor transport behavior possibly even worse than the HHs. Any approach of developing Si-based QCLs based on transitions between conduction subbands needs to necessarily go beyond the conventional methods in selecting the material system and growth technique.

Prospects of developing such Si-based QCLs have been investigated theoretically. One approach stayed with the Si-rich SiGe/Si material system but instead of the conventional growth direction in (100), the structural orientation has been rotated to the [111] crystal plane [61]. Conduction band offset was calculated to be $160 \mathrm{meV}$ at the conduction band minima consisting of six-degenerate $\Delta$-valleys, sufficient for designing far IR QCLs. The effective mass along (111) direction can be obtained as the geometric average of the longitudinal and transverse effective masses of the $\Delta$-valley, $\sim 0.26 m_{0}$, lower than that of longitudinal $m_{l} \sim 0.9 m_{0}$ in the (100) structure. Another design relying on the Ge-rich $\mathrm{Ge} / \mathrm{SiGe}$ material system has been proposed to construct conduction band QCLs using compressively strained Ge QWs and tensile strained $\mathrm{Si}_{0.22} \mathrm{Ge}_{0.78}$ alloy barriers grown on a relaxed [100] $\mathrm{Si}_{1-y} \mathrm{Ge}_{\mathrm{y}}$ buffer [62]. The intersubband transitions in this design are within the $L$-valleys which are the conduction band minima in Ge QWs whose effective mass along (100) direction has been determined to be $\sim 0.12 m_{0}$. Since $\mathrm{Si}_{1-\mathrm{x}} \mathrm{Ge}_{\mathrm{x}}$ alloys with $x<0.85$ are similar to $\mathrm{Si}$ in that the conduction band minima appear in the $\Delta$-valleys, the conduction band lineup in the $\mathrm{Ge} / \mathrm{Si}_{0.22} \mathrm{Ge}_{0.78}$ structure is rather complex with conduction band minima in Ge at $L$-valleys but in $\mathrm{Si}_{0.22} \mathrm{Ge}_{0.78}$ at $\Delta_{2}$-valleys along the (100) growth direction. Although the band offset at the $L$-valleys is estimated to be as high as $138 \mathrm{meV}$, the overall band offset between the absolute conduction band minima in $\mathrm{Ge}$ and $\mathrm{Si}_{0.22} \mathrm{Ge}_{0.78}$ is only $41 \mathrm{meV}$. Although the quantum confinement effect helps to lift those electron subbands at $\Delta_{2}$-valleys, the two $\Delta_{2}$-valleys are inevitably entangled with the $L$-valleys in the conduction band, leading to design complexity and potentially creating additional nonradiative decay channels for the upper laser state.

Recently, a new group-IV material system that expands beyond the $\mathrm{Si}_{1-x} \mathrm{Ge}_{x}$ alloys has been successfully demonstrated with the incorporation of $\mathrm{Sn}$. These new ternary $\mathrm{Ge}_{1-x-y} \mathrm{Si}_{x} \mathrm{Sn}_{\mathrm{y}}$ alloys have been studied for the possibility of forming direct band gap semiconductors[6366]. Since the first successful growth of this alloy [67], device- quality epilayers with a wide range of alloy contents have been achieved [68,69]. Incorporation of Sn provides the opportunity to engineer separately the strain and band structure since we can vary the $\mathrm{Si}(\mathrm{x})$ and Sn (y) compositions independently. Certain alloy compositions of this material system offer three advantages: (1) the possibility of a "cleaner" conduction band lineup in which the $L$-valleys in both well and barrier sit below other valleys $(\Gamma, \Delta),(2)$ an electron effective mass along the (001) growth direction that is much lower than the HH mass, and (3) a strain free structure that is lattice matched to Ge. In addition, recent advances in the direct growth of Ge layer on Si provide a relaxed matching buffer layer on a Si substrate upon which the strain-free $\mathrm{Ge} / \mathrm{Ge}_{1-x-y} \mathrm{Si}_{x} \mathrm{Sn}_{\mathrm{y}}$ is grown [70]. Based on this material system, a strain free QCL operating in the conduction L-valleys was proposed [71].

Since band offsets between ternary Sn-containing alloys and $\mathrm{Si}$ or Ge are not known experimentally, we have calculated the conduction band minima for a lattice- matched heterostructure consisting of $\mathrm{Ge}$ and a ternary $\mathrm{Ge}_{1-x-y} \mathrm{Si}_{x} \mathrm{Sn}_{y}$ based on Jaros' band offset theory [72] which is in good agreement with experiment for many heterojunction systems. For example, this theory predicts an average valence band offset, $\Delta E_{v, a v}=0.48 \mathrm{eV}$ for a 
$\mathrm{Ge} / \mathrm{Si}$ heterostructure (higher energy on the Ge side), close to the accepted value of $\Delta E_{v, a v}=0.5 \mathrm{eV}$. The basic ingredients of our calculation are the average (between $\mathrm{HH}, \mathrm{LH}$, and SO bands) valence band offset between the two materials and the compositional dependence of the band structure of the ternary alloy. For the Ge/ $\alpha$-Sn interface, Jaros' theory predicts $\Delta E_{v, a v}=0.69 \mathrm{eV}$ (higher energy on the $\mathrm{Sn}$ side). For the $\mathrm{Ge}_{1-x-y} \mathrm{Si}_{x} \mathrm{Sn}_{y} / \mathrm{Ge}$ interface we have used the customary approach for alloy semiconductors, interpolating the average valence band offsets for the elementary heterojunctions $\mathrm{Ge} / \mathrm{Si}$ and $\mathrm{Ge} / \alpha-\mathrm{Sn}$. Thus we used (in eV)

$$
\Delta E_{v, a v}(x, y)=E_{v, a v}(\mathrm{GeSiSn})-E_{v, a v}(\mathrm{Ge})=0.48 x+0.69 y .
$$

Once the average valence band offset is determined, the energies of individual conduction band edges in the $\mathrm{Ge}_{1-x-y} \mathrm{Si}_{x} \mathrm{Sn}_{y}$ alloy can be calculated relative to those in Ge from the compositional dependence of the spin-orbit splitting of the top valence band states and the compositional dependence of the energy separations between those conduction band edges and the top of the valence band in the alloy [73]. We have assumed that all required alloy energies can be interpolated between the known values for $\mathrm{Si}, \mathrm{Ge}$, and a-Sn as

$$
\begin{aligned}
E_{\mathrm{GeSiSn}}(x, y)= & E_{\mathrm{GeSiSn}}(1-x-y)+E_{\mathrm{Si}} x+E_{\mathrm{Sn}} y \\
& -b_{\mathrm{GeSi}}(1-x-y) x-b_{\mathrm{GeSn}}(1-x-y) y-b_{\mathrm{SiSn}} x y
\end{aligned}
$$

The bowing parameters $b_{\mathrm{GeSi}}, b_{\mathrm{GeSn}}$, and $b_{\mathrm{SiSn}}$ have been discussed in Refs. [74] and [75]. Finally, for the indirect conduction band minimum near the $X$-point, Weber and Alonso find

$$
E_{x}=0.931+0.018 x+0.206 x^{2}
$$

(in $\mathrm{eV}$ ) for $\mathrm{Ge}_{1-x} \mathrm{Si}_{x}$ alloys [76]. On the other hand, the empirical pseudo-potential calculations of Chelikovsky and Cohen place this minimum at $0.90 \mathrm{eV}$ in $\alpha-\mathrm{Sn}$, virtually the same as its value in pure Ge [77]. We thus assume that the position of this minimum in ternary $\mathrm{Ge}_{1-x-y} \mathrm{Si}_{x} \mathrm{Sn}_{y}$ alloys is independent of the $\mathrm{Sn}$ concentration $y$. The conduction band minima results are shown in Fig. 11 for Sn concentrations $0<y<0.1$. The Si concentration $x$ was calculated using Vegard's law in such a way that the ternary $\mathrm{Ge}_{1-x-y} \mathrm{Si}_{x} \mathrm{Sn}_{y}$ is exactly lattice-matched with Ge.

It can be seen from Fig. 11 that a conduction-band offset of $150 \mathrm{meV}$ at $L$-valleys can be obtained between lattice-matched $\mathrm{Ge}$ and $\mathrm{Ge}_{0.76} \mathrm{Si}_{0.19} \mathrm{Sn}_{0.05}$ alloy while all other conductionband valleys $\left(\Gamma, X\right.$, etc) are above the $L$-valley band edge of the $\mathrm{Ge}_{0.76} \mathrm{Si}_{0.19} \mathrm{Sn}_{0.05}$ barrier. This band alignment presents a desirable alloy composition from which a QCL operating at Lvalleys can be designed using $\mathrm{Ge}$ as $\mathrm{QWs}$ and $\mathrm{Ge}_{0.76} \mathrm{Si}_{0.19} \mathrm{Sn}_{0.05}$ as barriers without the complexity arising from other energy valleys.

Figure 12 shows the QCL structure based upon $\mathrm{Ge} / \mathrm{Ge}_{0.76} \mathrm{Si}_{0.19} \mathrm{Sn}_{0.05} \mathrm{QWs}$. Only L-valley conduction-band lineups are shown in the potential diagram under an applied electric field of $10 \mathrm{kV} / \mathrm{cm}$. In order to solve the Schrödinger equation to yield subbands and their associated envelope functions, it is necessary to determine the effective mass $m_{z}^{*}$ along the (001) growth direction $(z)$ within the constant-energy ellipsoids at the L-valleys along the (111) direction which is tilted with respect to (100). Using the $L$-valley principal transverse effective mass $m_{t}^{*}=0.08 m_{0}$, and the longitudinal effective mass $m_{l}^{*}=1.60 m_{0}$ for Ge, we obtain $m_{z}^{*}=\left(2 / 3 m_{t}^{*}+1 / 3 m_{l}^{*}\right)^{-1}=0.12 m_{0}$. The squared magnitudes of all envelope functions are plotted at energy positions of their associated subbands. As shown in Fig. 12, each period of the QCL has an active region for lasing emission and an injector region for 
carrier transport. These two regions are separated by a $30 \AA$ barrier. The active region is constructed with 3 coupled Ge QWs that give rise to three subbands marked 1, 2, and 3. The lasing transition at the wavelength of $49 \mu \mathrm{m}$ is between the upper laser state 3 and the lower laser state 2. The injector region consists of $4 \mathrm{Ge}$ QWs of decreasing well widths all

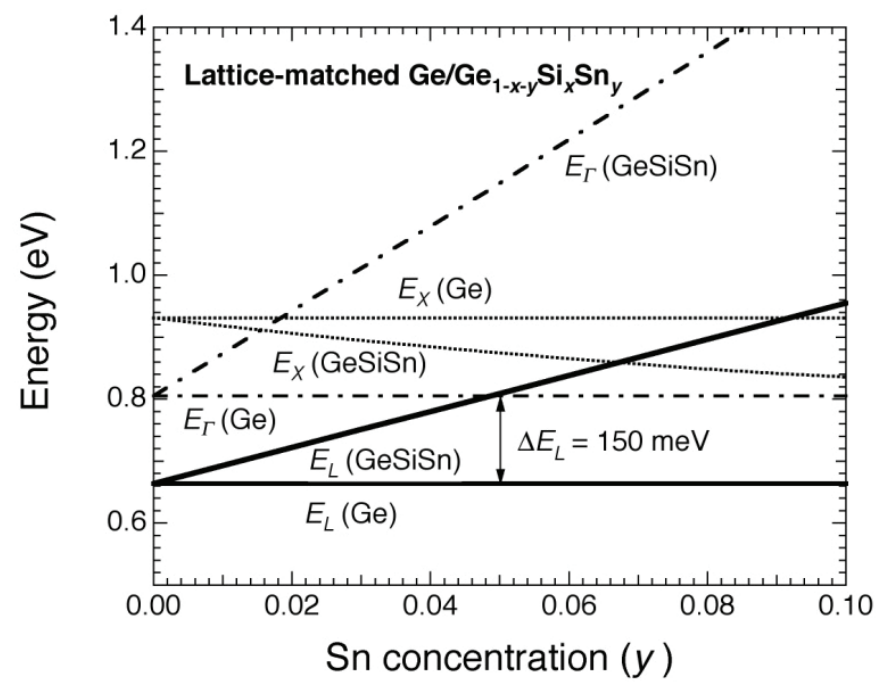

Fig. 11. Conduction band minima at $L, \Gamma, X$ points of $\mathrm{Ge}_{1-x-y} \mathrm{Si}_{x} \mathrm{Sn}_{y}$ that is lattice matched to Ge [71].

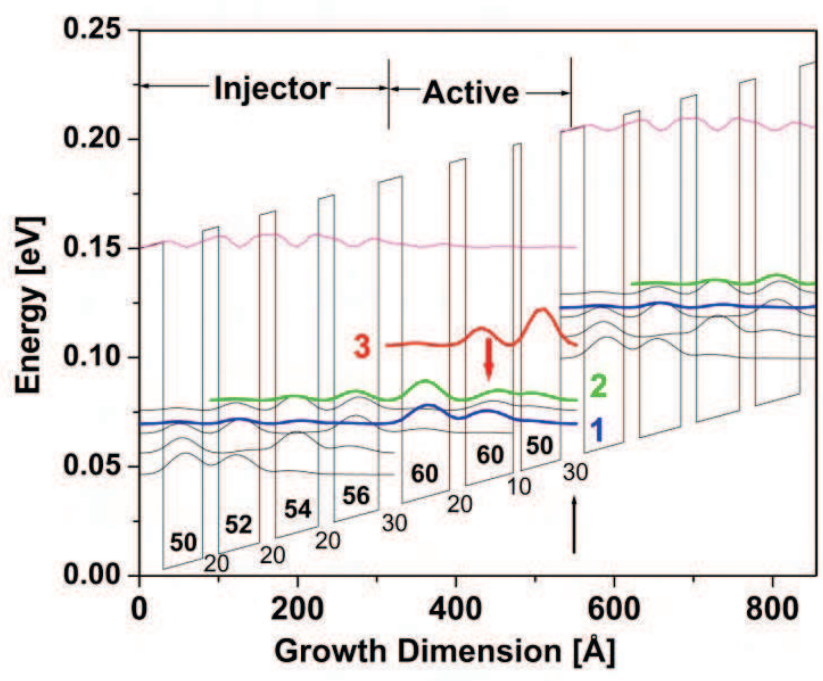

Fig. 12. $L$-valley conduction band profile and squared envelope functions under an electric field of $10 \mathrm{kV} / \mathrm{cm}$. Layer thicknesses in angstrom are marked with bold numbers for Ge QWs and regular for GeSiSn barriers. Array marks the injection barrier [71]. 
separated by $20 \AA \mathrm{Ge}_{0.76} \mathrm{Si}_{0.19} \mathrm{Sn}_{0.05}$ barriers. The depopulation of lower state 2 is through scattering to state 1 and to the miniband downstream formed in the injector region. These scattering processes are rather fast because of the strong overlap between the involved states. Another miniband in the injector region formed of quasi-bound states is situated 45 $\mathrm{meV}$ above the upper laser state 3, effectively preventing escape of electrons from upper laser state 3 into the injector region.

The nonradiative transition rates between different subbands in such a low-doped nonpolar material system with low injection current should be dominated by deformation-potential scattering of nonpolar optical and acoustic phonons. For this Ge-rich structure, we have used bulk-Ge phonons for calculation of the scattering rate to yield lifetimes for the upper laser state $\tau_{3}$ and the lower laser state $\tau_{2}$, as well as the $3 \rightarrow 2$ scattering time $\tau_{32}$ [78]. The results obtained from Eq.(38) are shown in Fig. 13 as a function of operating temperature. These lifetimes are at least one-order of magnitude longer than those of III-V QCLs owing to the nonpolar nature of GeSiSn alloys. The necessary condition for population inversion $\tau_{32}>\tau_{2}$ is satisfied throughout the temperature range. Using these predetermined lifetimes in the population rate equation under current injection:

$$
\left\{\begin{array}{l}
\frac{\partial N_{3}}{\partial t}=\frac{\eta J}{e}-\frac{N_{3}-\bar{N}_{3}}{\tau_{3}} \\
\frac{\partial N_{2}}{\partial t}=\frac{N_{3}-\bar{N}_{3}}{\tau_{32}}-\frac{N_{2}-\bar{N}_{2}}{\tau_{2}}
\end{array}\right.
$$

where $N_{i},(i=2,3)$ is the area carrier density per period in subband $i$ under injected current density $J$ with an injection efficiency $\eta$, and $\bar{N}_{i}$ is the area carrier density per period due to thermal population. Solving the above rate equation at steady state yields population inversion

$$
N_{3}-N_{2}=\tau_{3}\left(1-\frac{\tau_{2}}{\tau_{32}}\right) \frac{\eta J}{e}-\left(\bar{N}_{2}-\bar{N}_{3}\right)
$$

which can then be used to evaluate the optical gain of the TM polarized mode following Eq.(28) at the lasing transition energy $\hbar \omega_{0}=E_{3}-E_{2}=25 \mathrm{meV}$ as

$$
\gamma\left(\omega_{0}\right)=\frac{2 e^{2} m_{0}^{2} \omega_{0} z_{23}^{2}}{\varepsilon_{0} c n_{\text {eff }} m_{z}^{* 2} \Gamma L_{p}}\left[\tau_{3}\left(1-\frac{\tau_{2}}{\tau_{32}}\right) \frac{\eta J}{e}-\left(\bar{N}_{2}-\bar{N}_{3}\right)\right] .
$$

For the QCL structure in Fig. 12, the following parameters are used: index of refraction $n_{\text {eff }}=3.97$, lasing transition FWHM $\Gamma=10 \mathrm{meV}$, length of one period of the QCL $L_{p}=532 \AA$, area doping density per period of $10^{10} \mathrm{~cm}^{2}$, and unit injection efficiency $\eta=1$.

Since the relatively small conduction band offset limits the lasing wavelength to the far-IR or $\mathrm{THz}$ regime (roughly $30 \mu \mathrm{m}$ and beyond), the waveguide design can no longer rely on that of conventional dielectric waveguides such as those used in laser diodes and mid IR QCLs. This is mainly because the thickness required for the dielectric waveguide would have exceeded what can be realized with the epitaxial techniques employed to grow the laser structures. One solution is to place the QCL active structure between two metal layers to form the so-called plasmon waveguide $[79,80]$. While the deposition of top metal is trivial, placing bottom metal requires many processing steps such as substrate removal, metal 
deposition, and subsequent wafer bonding. The QCL waveguides are typically patterned into ridges as shown in Fig. 14.

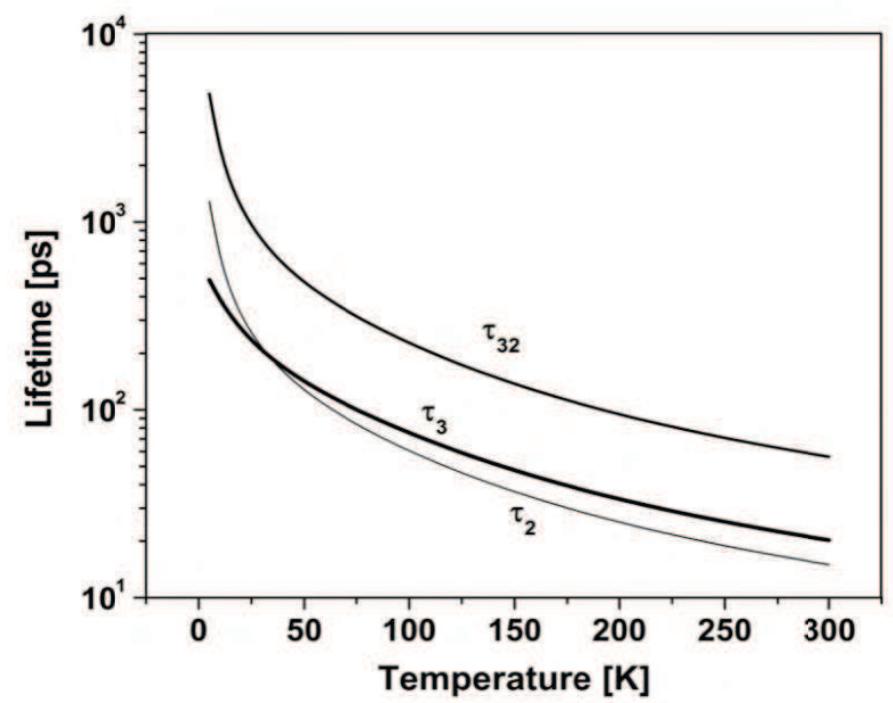

Fig. 13. Upper state lifetime $\tau_{3}$, lower state lifetime $\tau_{2}$, and scattering time $\tau_{32}$ between them as a function of temperature.

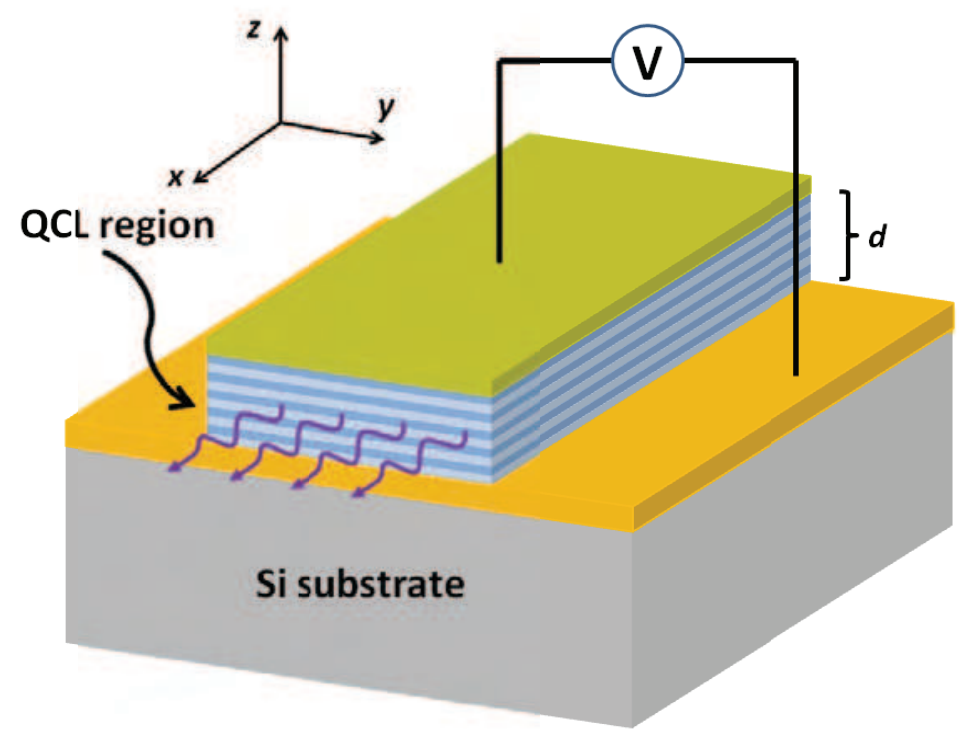

Fig. 14. Schematic of a ridge plasmon waveguide with the Ge/GeSiSn QCL sandwiched between two metal layers. 
This plasmon waveguide supports only TM polarized EM mode that is highly confined within the QCL region, $-d / 2<z<d / 2$. We can assume Drude model to describe the metal dielectric function

$$
\varepsilon_{M}=1-\frac{\omega_{p}^{2}}{\omega^{2}+j \gamma_{m} \omega}
$$

where $\omega_{p}$ is the metal plasmon frequency, and $\gamma_{m}$ is the metal loss $\left(\hbar \omega_{p}=8.11 \mathrm{eV}, \hbar \gamma_{m}=\right.$ $65.8 \mathrm{meV}$ for $\mathrm{Au}[81])$, and $\varepsilon_{D}=n_{\text {eff }}^{2} \approx 16$ for the Ge rich $\mathrm{Ge} / \mathrm{Ge}_{0.76} \mathrm{Si}_{0.19} \mathrm{Sn}_{0.05}$ QCL active region. Consider the EM wave propagate along the $x$-direction as shown in Fig. 14, its electric field can then be obtained as

$$
E=\left\{\begin{array}{rr}
\frac{E_{0}}{\varepsilon} \cosh (k d / 2)(j \beta \hat{\mathbf{z}}+q \widehat{\boldsymbol{x}}) e^{-q(z-d / 2)} e^{j(\beta x-\omega t)}, & z>\frac{d}{2} \\
E_{0}[j \beta \cosh (k z) \hat{\mathbf{z}}-k \sinh (k z) \widehat{\boldsymbol{x}}] e^{j(\beta x-\omega t)}, & |z|<\frac{d}{2} \\
\frac{E_{0}}{\varepsilon} \cosh (k d / 2)(j \beta \hat{\mathbf{z}}-q \widehat{\boldsymbol{x}}) e^{q(z+d / 2)} e^{j(\beta x-\omega t)}, & z<-\frac{d}{2}
\end{array}\right.
$$

where $\varepsilon=\varepsilon_{M} / \varepsilon_{D}$, and $E_{0}$ is a constant. The complex propagation constant $\beta=\beta^{\prime}+j \beta^{\prime \prime}$ follows these relations, $\beta^{2}-k^{2}=k_{D}^{2}, \beta^{2}-q^{2}=\varepsilon k_{D}^{2}$ with $k_{D}=\sqrt{\varepsilon_{D}} \omega / c$. It is easy to see that the continuity of the normal component of the electric displacement is satisfied at the boundaries $z= \pm d / 2$, the requirement of continuity of tangential electric field leads to

$$
k^{2}\left[\varepsilon^{2} \tanh ^{2}\left(\frac{k d}{2}\right)-1\right]=k_{D}^{2}(1-\varepsilon)
$$

which determines the TM modes that can propagate in this plasmon waveguide. The waveguide loss $\alpha_{w}$ is dominated by the metal loss, which can be determined from the imaginary part of the propagation constant, as $\alpha_{w}=2 \beta "$. As a superposition of two surface plasmon modes bound to the two metal-dielectric interfaces at $z= \pm d / 2$, this TM mode decays exponentially into the metal, providing an excellent optical confinement factor defined as $\Gamma_{w}=\int_{-d / 2}^{d / 2}|E|^{2} d z / \int_{-\infty}^{\infty}|E|^{2} d z$. We have simulated the TM-polarized mode in a QCL structure of 40 periods $(d=2.13 \mu \mathrm{m})$ that is confined by double-Au-plasmon waveguide and obtained near unity optical confinement $\Gamma_{w} \approx 1.0$ and waveguide loss $\alpha_{w}=110 / \mathrm{cm}$. Assuming a mirror loss $\alpha_{m}=10 / \mathrm{cm}$ for a typical cavity length of $1 \mathrm{~mm}$, the threshold current density $J_{t h}$ can be calculated from the balancing relationship, $\Gamma_{w} \gamma_{t h}=$ $\alpha_{w}+\alpha_{m}$, where $\gamma_{t h}$ is the optical gain Eq.(44) obtained at the threshold $J_{t h}$. The result is shown in Fig. 15 for $J_{t h}$ that ranges from $22 \mathrm{~A} / \mathrm{cm}^{2}$ at $5 \mathrm{~K}$ to $550 \mathrm{~A} / \mathrm{cm}^{2}$ at $300 \mathrm{~K}$. These threshold values are lower than those of III-V QCLs as a result of the longer scattering times due to nonpolar optical phonons.

While GeSiSn epilayers with alloy compositions suitable for this QCL design have been grown with MOCVD [68,69], implementation of Ge/GeSiSn QCLs is currently challenged by the structural growth of the large number of hetero-layers in the QCL structure with very fine control of layer thicknesses and alloy compositions. Nevertheless, progress is being made towards experimental demonstration. 


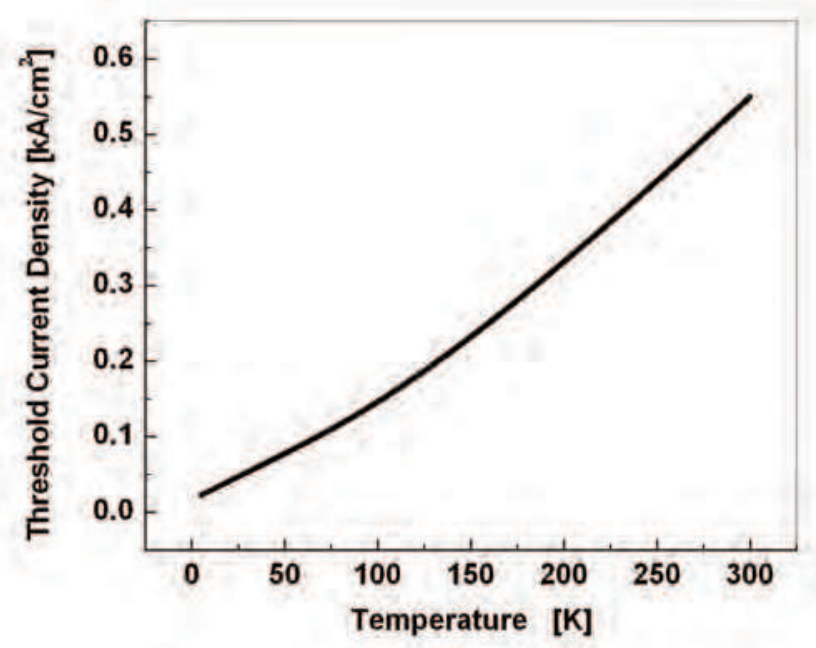

Fig. 15. Simulated threshold current density of the Ge/GeSiSn QCL as a function of temperature.

\section{Summary}

Silicon-based lasers have been long sought after for the possibility of monolithic integration of photonics with high-speed Si electronics. Many parallel approaches are currently taken to reach this goal. Among them Si nanocrystals and Er-doped Si have been investigated rather extensively. While EL has been demonstrated, lasing has not been observed. The only reported lasing in Si so far has been achieved using stimulated Raman scattering which requires optical pumping at very high intensity on a device of large scale - impractical for integration with Si electronics. The QCLs that have been successfully developed in III-V semiconductors offer an important alternative for the development of Si-based lasers. The salient feature of QCLs is that lasing transitions take place between subbands that are within the conduction band without crossing the band gap. Such a scheme makes the indirect nature of the Si band gap irrelevant. In order to appreciate the QCL designs, some theoretical background underlying the basic operating principles has been introduced here. In particular, subband formation and energy dispersion in semiconductor QWs are described in the framework of envelope functions with the effective-mass approximation for both conduction and valence band taking into account mixing between $\mathrm{HH}, \mathrm{LH}$, and $\mathrm{SO}$ bands. Optical gain based on ISTs is derived and intersubband lifetimes are discussed with a more detailed treatment of carrier-phonon scattering.

The development of Si-based QCLs has been primarily focused on ISTs between valence subbands in the Si-rich SiGe/Si material system. Such a material system has been routinely used in CMOS-compatible processes. There are two reasons for using holes instead of electrons. One is that the compressively strained $\mathrm{Si}_{1-x} \mathrm{Ge}_{\mathrm{x}}$ with tensile strained $\mathrm{Si}$ grown on a relaxed $\mathrm{Si}_{1-y} \mathrm{Ge}_{\mathrm{y}}$ has very small conduction band offset - QWs are too shallow to allow for elaborate QCLs. Tensile strained $\mathrm{Si}_{1-\mathrm{x}} \mathrm{Ge}_{\mathrm{x}}$, on the other hand, can have larger conduction 
band offset, but the conduction band minima occur at the two $\Delta_{2}$-valleys whose effective mass (longitudinal) along the growth direction is heavy $\left(m_{l} \sim 0.9 m_{0}\right)$ resulting in small oscillator strength and poor transport behavior such as reduced tunneling probabilities. It is generally believed that SiGe QCLs have to be pursued within the valence band as a p-type device. But the situation in valence band also presents challenges in several perspectives. First, the strong mixing of $\mathrm{HH}, \mathrm{LH}$, and SO bands makes the QCL design exceedingly cumbersome albeit the opportunities presented by the strong nonparabolicity in valence subbands to take advantage of schemes such as the inverted effective mass where the total population inversion between subbands may not be necessary. Second, there is a great deal of uncertainty in various material parameters for the SiGe alloy - often times approximation has to be made to linearly extrapolate parameters from those of $\mathrm{Si}$ and $\mathrm{Ge}$, thus, the accuracy of the designs has a great degree of ambiguity. Third, any valence QCLs have no choice but to deal with $\mathrm{HH}$ subbands; their large effective mass hinders carrier injection efficiency and leads to small IST oscillator strength between laser states. Fourth, for any significant band offset needed to implement QCLs, lattice-mismatch-induced strain in SiGe QWs and $\mathrm{Si}$ barriers even in strain balanced structures is significant, which presents a challenge in structural growth and device processing. While EL was demonstrated from a valence-band $\mathrm{SiGe} / \mathrm{Si}$ quantum cascade emitter nearly a decade ago, lasing remains elusive. Recently, several ideas of developing Si-based conduction-band QCLs have emerged to circumvent the hurdles in the SiGe/Si valence-band approach. The proposals offer ways to increase the conduction band offset and to reduce the effective mass along the growth direction. One scheme proposes to orient the structural growth along the (111) direction, and another relies on ISTs in the L-valleys of the conduction band in Ge-rich Ge/SiGe material system. The former has accomplished more in increasing the conduction-band offset, and the latter in reducing the effective mass. A third approach that expands the material system beyond SiGe to GeSiSn has been discussed in detail. A Ge/Ge $\mathrm{G}_{0.76} \mathrm{Si}_{0.19} \mathrm{Sn}_{0.05}$ QCL that operates at $L$-valleys of the conduction band was designed. According to our estimation of the band lineup, this particular alloy composition gives a "clean" conduction band offset of $150 \mathrm{meV}$ at $L$-valleys with all other energy valleys conveniently out of the way. All QCL layers are lattice matched to a Ge buffer layer on a Si substrate and the entire structure is therefore strain free. The electron effective mass along the growth direction is much lighter than that of heavy holes bringing a significant improvement in tunneling rates and oscillator strengths. The lasing wavelength of this device is $49 \mu \mathrm{m}$. With different GeSiSn alloy compositions that are lattice matched to Ge, QCLs can be tuned to lase at other desired wavelengths. Lifetimes determined from the deformation potential scattering of nonpolar optical and acoustic phonons are at least an order of magnitude longer than those in III-V QCLs with polar optical phonons, leading to a reduction in threshold current density and the possibility of room temperature operation. While there are considerable challenges in material growth of this QCL design, advances in fine control of structural parameters including layer thicknesses and alloy compositions are moving towards implementation of conduction-band QCLs in the GeSiSn system.

When are we going to realize Si-based lasers that can be integrated with Si electronics? Clearly, breakthroughs in material science and device innovation are necessary before that happens, but with the variety of approaches that are being pursued--driven by the potential pay-off in commercialization--the prospect is promising. 


\section{References}

[1] R. A. Soref and J. P. Lorenzo, "All-silicon active and passive guided-wave components for $\lambda=1.3$ and 1.6 $\mu \mathrm{m}$," IEEE Journal of Quantum Electronics QE-22, (1986) 873-879.

[2] R. A. Soref, "Silicon-based optoelectronics," Proceedings of the IEEE 81, (1993) 1687-1706.

[3] L.Pavesi, L. Dal Negro, C. Mazzoleni, G. Franzo, and F. Priolo, "Optical gain in silicon nanocrystals," Nature, 408, (2000) 440-444.

[4] J. Ruan, P. M. Fauchet, L. Dal Negro, C. Mazzoleni, and L.Pavesi, "Stimulated emission in nanocrystalline silicon superlattice," Applied Physics Letters 83, (2003) 5479-5481.

[5] M. J. Chen, J. L. Yen, J. Y. Li, J. F. Chang, S. C. Tsai, and C. S. Tsai, “Stimulated emission in a nanostructured silicon pn-junction diode using current injection," Applied Physics Letters 84, (2004) 2163-2165.

[6] E. Desurvire, Erbium-doped Fiber Amplifiers: Principles and Applications, Wiley, New York, (1994)

[7] G. Franzò and S. Coffa, "Mechanism and performance of forward and reverse bias electroluminescence at $1.54 \mu \mathrm{m}$ from Er-doped Si diodes," Journal of Applied Physics 81, (1997) 2784-2793.

[8] F. Priolo, G. Franzò, S. Coffa and A. Carnera, "Excitation and nonradiative deexcitation processes of $\mathrm{Er}^{3+}$ in crystalline Si," Physical Review B 57, (1998) 4443-4455.

[9] A. J. Kenyon, P. F. Trwoga, M. Federighi, and C W Pitt “Optical properties of PECVD erbium-doped silicon-rich silica: evidence for energy transfer between silicon microclusters and erbium ions," Journal of Physics: Condensed Matter 6, (1994) L319324.

[10] M. E. Castagna, S. Coffa, L. carestia, A. Messian, and C. Buongiorno, "Quantum dot materials and devices for light emission in silicon," Proceedings of 32nd European Solid-State Device Research Conference (ESSDERC 2002), Fireze, Italy, (2002) D21.3

[11] R. Calps, D. Dimitropoulos, V. Raghunathan, Y. Han, and B. Jalali, "Observation of stimulated Raman scattering in silicon waveguides," Optics Express 11, (2003) 17311739

[12] A. Liu, H. Rong, M. Paniccia, O. Cohen, D. Hak, “Net optical gain in a low loss siliconon-insulator waveguide by stimulated Raman scattering," Optics Express 12, (2004) 4261-4268.

[13] O. Boyraz and B. Jalali, "Demonstration of a silicon Raman laser," Optics Express 12, (2004) 5269-5273.

[14] B. Jalali, "Silicon photonics," Journal of Lightwave Technology 24, (2006) 4600-4615.

[15] H. Park, A. W. Fang, S. Kodama, and J. E. Bowers, "Hybrid silicon evanescent laser fabricated with a silicon waveguide and III-V offset quantum wells," Optics Express 13, (2005) 9460-9464.

[16] J. H. Park and A. J. Steckl, “Demonstration of a visible laser on silicon using Eu-doped GaN thin films," Journal of Applied Physics 98, (2005) 056108

[17] Z. Mi, P. Bhattacharya, J. Yang, and K. P. Pipe, "Room-temperature self-organized $\mathrm{In}_{0.5} \mathrm{Ga}_{0.5}$ As quantum dot laser on silicon," Electronics Letters 41, (2005) 742-744.

[18] L. Esaki and R. Tsu, "Superlattice and negative differential conductivity in semiconductors", IBM Journal of Research and Development 14, (1970), 61-65.

[19] R. F. Kazarinov and R. Suris, "Electric and electromagnetic properties of semiconductors with a superlattice," Soviet Physics - Semiconductors 5, (1971) 797. 
[20] J. Faist, F. Capasso, D. L. Sivco, A. L. Hutchinson, C. Sirtori, and A. Y. Cho, "Quantum cascade laser," Science 264, (1994) 553-556

[21] G. Sun and J. B. Khurgin, "Feasibility of optically pumped four-level infrared lasers," in Intersubband Transitions in Quantum Wells, (Edited by E. Rosencher, B. Vinter, and B. Levine, Plenum Press, New York, 1992), pp.219-226

[22] G. Sun and Khurgin, "Optically pumped four-level infrared laser based on intersubband transitions in multiple quantum wells: feasibility study," IEEE Jounral of Quantum Electronics QE-29, (1993) 1104-1111.

[23] D. G. Revin, J. W. Cockburn, M. J. Steer, R. J. Airey, M. Hopkinson, A. B. Krysa, L. R. Wilson, and S. Menzel, "InGaAs/AlAsSb/InP quantum cascade lasers operating at wavelengths close to $3 \mu \mathrm{m}$," Applied Physics Letters 90, (2007) 021108.

[24] M. P. Semtsiv, M. Wienold, S. Dressler, and W. T. Masselink, "Short-wavelength $(\lambda \approx 3.05 \mu \mathrm{m})$ InP-based strain-compensated quantum-cascade laser," Applied Physics Letters 90, (2007) 051111.

[25] R. Colombelli, F. Capasso, C. Gmachl, A. L. Hutchinson, D. L. Sivco, A. Tredicucci, M. C. Wanke, A. M. Sergent, and A. Y. Cho, "Far-infrared surface-plasmon quantumcascade lasers at $21.5 \mu \mathrm{m}$ and $24 \mu \mathrm{m}$ wavelengths," Applied Physics Letters 78, (2001) 2620-2622.

[26] Y. Bai, S. Slivken, S. R. Darvish, and M. Razeghi, "Room temperature continuous wave operation of quantum cascade lasers with $12.5 \%$ wall plug efficiency," Applied Physics Letters 93, (2008) 021103.

[27] A. Lyakh, C. Pflügl, L. Diehl, Q. J. Wang, F. Capasso, X. J. Wang, J. Y. Fan, T. TanbunEk, R. Maulini, A. Tsekoun, R. Go, and C. Kumar N. Patel, "1.6 W high wall plug efficiency, continuous-wave room temperature quantum cascade laser emitting at $4.6 \mu \mathrm{m}, "$ Applied Physics Letters 92, (2008) 111110.

[28] B. S. Williams, “Tereahertz quantum-cascade lasers," Nature Phtonics 1, (2007) 517-525.

[29] S. R. White and L. J. Sham, "Electronic properties of flat-band semiconductor heterostructures," Physical Review Letters 47, (1981) 879 - 882.

[30] E. O. Kane, "Band structure of indium antimonide," Journal Physics and Chemistry of Solids 1, (1957) 249-261.

[31] G. Bastard, Wave Mechanics Applied to Semiconductor Heterostructures (Les Editions de Physique, les Ulis), 1998, Chapter 3.

[32] J. M. Luttinger and W. Kohn, "Motion of Electrons and Holes in Perturbed Periodic Fields," Physical Review 97, (1955) 869-883.

[33] J. M. Luttinger, "Quantum Theory of Cyclotron Resonance in Semiconductors: General Theory," Physical Review 102, (1956) 1030-1041.

[34] G. L. Bir and G. E. Pikus, Symmetry and Strain-Induced Effects in Semiconductors (Wiley, New York, 1974)

[35] G. Sun, Y. Lu, J. B. Khurgin, "Valence intersubband lasers with inverted light-hole effective mass," Applied Physics Letters 72, (1998) 1481-1483.

[36] L. C. L. Y. Voon and L. R. Ram-Mohan, "Tight-binding representation of the optical matrix elements: theory and applications," Physical Review B 47, (1993) 1550015508.

[37] L. C. West and S. J. Eglash, "First observation of an extremely large-dipole infrared transition within the conduction band of a GaAs quantum well," Applied Physics Letters 46, (1985) 1156-1158. 
[38] G. Sun, L. Friedman, and R. A. Soref, "Intersubband lasing lifetimes of SiGe/Si and GaAs/AlGaAs multiple quantum well structures," Applied Physics Letters 66, (1995) 3425-3427.

[39] B. K. Ridley, “The electron-phonon interaction in quasi-two-dimensional semiconductor quantum-well structures," Journal of Physics C: Solid state Physics 15, (1982) 58995917.

[40] P. J. Price, "Two-dimensional electron transport in semiconductor layers. I. Phonon scattering," Annals of Physics 133, (1981) 217-239.

[41] P. Harrison, Quantum Wells, Wires, and Dots, (John Wiley \& Sons, 2000) p.272

[42] H. Callebaut, S. Kumar, B.S. Williams, Q. Hu, and J.L. Reno, "Importance of electron impurity scattering for electron transport in terahertz quantum-cascade lasers," Applied Physics Letters 84, (2004) 645-647.

[43] R. Ferreira and G. Bastard, "Evaluation of some scattering times for electrons in unbiased and biased single-and multiple-quantum-well structures," Physical Review B 40, (1989) 1074-1086.

[44] M. Hartig, J. D. Ganière, P. E. Selbmann, B. Deveaud, and L. Rota, “Density dependence of carrier-carrier induced intersubband scattering in GaAs/AlGaAs quantum wells," Physical Review B 60, (1999) 1500-1503.

[45] J. D. Cressler, SiGe and Si Strained-Layer Epitaxy for Silicon Heterostructure Devices, CRC Press, (2008)

[46] L. Friedman, R. A. Soref, G. Sun, and Y. Lu, “Theory of the strain-symmetrized siliconbased Ge-Si superlattice laser," IEEE Journal of Selected Topics in Quantum Electronics 4, (1998) 1029-1034.

[47] L. Friedman, R. A. Soref, G. Sun, and Y. Lu, “Asymmetric strain-symmetrized Ge-Si interminiband laser," IEEE Photonics Technology Letters 10, (1998) 1715-1717.

[48] D. J. Paul, G. Matman, L. Lever, Z. Ikonić, R. W. Kelsall, D. Chrastina, G. Isella, H. von Känel, E. Müller, and A. Neels, "Si/SiGe bound-to-continuum terahertz quantum cascade emitters," ECS Transactions. 16, (2008) 865-874.

[49] J. Faist, F. Capasso, C. Sirtori, D. L. Sivco, A. L. Hutchinson, M. S. Hybertson, and A. Y. Cho, "Quantum cascade lasers without intersubband population inversion," Physical Review Letters 76, (1996) 411-414.

[50] L. Friedman, G. Sun, and R. A. Soref, "SiGe/Si THz laser based on transitions between inverted mass light-hole and heavy-hole subbands," Applied Physics Letters 78, (2001) 401-403.

[51] R. A. Soref and G. Sun, "Terahertz gain in SiGe/Si quantum staircase utilizing the heavy-hole inverted effective mass," Applied Physics Letters 79, (2001) 3639-3641.

[52] A. Fasolino, E. Molinari, and J. C. Maan, "Resonant quasiconfined optical phonons in semiconductor superlattices," Physical Review B 39, (1989) 3923-3926.

[53] G. Dehlinger, L. Diehl, U. Gennser, H. Sigg, J. Faist, K. Ensslin, D. Grützmacher, and E. Müller, "Intersubband electroluminescence from silicon-based quantum cascade structures," Science 290, (2000) 2277.

[54] I. Bormann, K. Brunner, S. Hackenbuchner, G. Zindler, G. Abstreiter, S. Schmult, and W. Wegscheider, "Midinfrared intersubband electroluminescence of Si/SiGe quantum cascade structures," Applied Physics Letters 80, (2002) 2260-2262.

[55] S. A. Lynch, R. Bates, D. J. Paul, D. J. Norris, A. G. Cullis, Z. Ikonić, R. W. Kelsall, P. Harrison, D. D. Arnone, and C. R. Pidgeon, "Intersubband electroluminescence 
from Si/SiGe cascade emitters at terahertz frequencies," Applied Physics Letters 81, (2002) 1543-1545.

[56] R. Bates, S. A. Lynch, D. J. Paul, Z. Ikonic, R. W. Kelsall, P. Harrison, S. L. Liew, D. J. Norris, A. G. Cullis, W. R. Tribe, and D. D. Arnone, "Interwell intersubband electroluminescence from Si/SiGe quantum cascade emitters," Applied Physics Letters 83, (2003) 4092-4094.

[57] J. Faist, M. Beck, T. Aellen, and E. Gini, "Quantum-cascade lasers based on a bound-tocontinuum transition," Applied Physics Letters 78, (2001) 147-149.

[58] G. Scalar, et al "Far infrared $(\lambda \cong 87 \mu \mathrm{m})$ bound-to-continuum quantum-cascade lasers operating up to 90K," Applied Physics Letters 82, (2003) 3165-3167.

[59] L. Diehl, S. Mentese, E. Müller,, D. Grützmacher, H. Sigg, U. Gennser, I. Sagnes, Y. Campiedelli, O. Kermarrec, D. Bensahel, and J. Faist, "Electroluminescence from strain-compensated $\mathrm{Si}_{0.2} \mathrm{Ge}_{0.8} / \mathrm{Si}$ quantum cascade structures based on a bound-tocontinuum transitions," Applied Physics Letters 81, (2002) 4700-4702.

[60] D. J. Paul, G. Matmon, L. Lever, Z. Ikonić, R. W. Kelsall, D. Chrastina, G. Isella, and H. von Känel, "Si/SiGe bound-to-continuum quantum cascade terahertz emitters," Proceedings of SPIE 6996, (2008) 69961C.

[61] L. Lever, A. Valavanis, Z. Ikonić, and R. W. Kelsall, "Simulated [111] Si-SiGe THz quantum cascade laser," Applied Physics Letters 92, (2008) 021124.

[62] K. Driscoll and R. Paiella, "Silicon-based injection lasers using electrical intersubband transitions in the L-valleys," Applied Physics Letters 89, (2006) 191110.

[63] R. A. Soref and C. H. Perry, "Predicted band gap of the new semiconductor SiGeSn," Journal of Applied Physics 69, (1991) 539-541.

[64] D. W. Jenkins and J. D. Dow, "Electronic properties of metastable Gex $\mathrm{Sn}_{1-x}$ alloys," Physical Review B 36, (1987) 7994-8000.

[65] G. He and H. A. Atwater, "Interband Transitions in $S n_{x} G e_{1-x}$ Alloys," Physical Review Letters 79, (1997) 1937-1940.

[66] J. Menéndez and J. Kouvetakis, "Type-I Ge/Ge $\mathrm{Ge}_{1-x-y} \mathrm{Si}_{x} \mathrm{Sn}_{y}$ strained-layer heterostructures with a direct Ge bandgap," Applied Physics Letters 85, (2004) 1175-1177.

[67] M. Bauer, C. Ritter, P. A. Crozier, J. Ren, J. Menéndez, G. Wolf, and J. Kouvetakis, "Synthesis of ternary SiGeSn semiconductors on $\mathrm{Si}(100)$ via $\mathrm{Sn}_{x} \mathrm{Ge}_{1-x}$ buffer layers," Applied Physics Letters 83, (2003) 2163-2165.

[68] R. Roucka, J. Tolle, C. Cook, A. V. G. Chizmeshya, J. Kouvetakis, V. D'Costa, J. Menendez, Zhihao D. Chen, and S. Zollner, "Versatile buffer layer architectures based on $\mathrm{Ge}_{1-x} \mathrm{Sn}_{x}$ alloys," Applied Physics Letters 86, (2005) 191912.

[69] P. Aella, C. Cook, J. Tolle, S. Zollner, A. V. G. Chizmeshya, and J. Kouvetakis, "Optical and structural properties of $\mathrm{Si}_{x} \mathrm{Sn}_{y} \mathrm{Ge}_{1-x-y}$ alloys," Applied Physics Letters 84, (2004) 888-890.

[70] M. A. Wistey, Y.-Y. Fang, J. Tolle, A. V. G. Chizmeshya, and J. Kouvetakis, “Chemical routes to $\mathrm{Ge} / \mathrm{Si}(100)$ structures for low temperature Si-based semiconductor applications," Applied Physics Letters 90, (2007) 082108.

[71] G. Sun, H. H. Cheng, J. Menéndez, J. B. Khurgin, and R. A. Soref, "Strain-free Ge/GeSiSn quantum cascade lasers based on L-valley intersubband transitions," Applied Physics Letters 90, (2007) 251105.

[72] M. Jaros, "Simple analytic model for heterojunction band offsets," Physical Review B 37, (1988) 7112-7114. 
[73] C. G. Van de Walle, "Band lineups and deformation potentials in the model-solid theory," Physical Review B 39, (1989) 1871-1883.

[74] V. R. D'Costa, C. S. Cook, A. G. Birdwell, C. L. Littler, M. Canonico, S. Zollner, J. Kouvetakis, and J. Menéndez, "Optical critical points of thin-film $\mathrm{Ge}_{1-y} \mathrm{Sn}_{y}$ alloys: A comparative $\mathrm{Ge}_{1-y} \mathrm{Sn}_{y} / \mathrm{Ge}_{1-x} \mathrm{Si}_{x}$ study," Physical Review B 73, (2006) 125207.

[75] V. R. D'Costa, C. S. Cook, J. Menéndez, J. Tolle, J. Kouvetakis, and S. Zollner, "Transferability of optical bowing parameters between binary and ternary groupIV alloys," Solid State Communications 138, (2006) 309-313.

[76] J. Weber and M. I. Alonso, "Near-band-gap photoluminescence of Si-Ge alloys," Physical Review B 40, (1989) 5683-5693.

[77] M. L. Cohen and T. K. Bergstresser, "Band Structures and Pseudopotential Form Factors for Fourteen Semiconductors of the Diamond and Zinc-blende Structures," Physical Review 141, (1966) 789-796.

[78] B. K. Ridley, Electrons and Phonons in semiconductor Multiplayers (Cambridge University Press, Cambridge, 1997), Chapter 1.

[79] B.S. Williams, S. Kumar, H. Callebaut, Q. Hu, and J.L. Reno," Terahertz quantum cascade laser at $\lambda_{\sim} 100 \mu \mathrm{m}$ using metal waveguide for mode confinement," Applied Physics Letters 83, (2003) 2124-2126.

[80] K. Unterrainer, R. Colombelli, C. Gmachl, F. Capasso, H.Y. Hwang, A.M. Sergent, D.L. Sivco, and A.Y. Cho, "Quantum cascade lasers with double metal-semiconductor waveguide resonators," Applied Physics Letters 80, (2002) 3060-3062.

[81] P. B. Johnson and R. W. Christy, "Optical Constants of the Noble Metals," Physical Review B 6, (1972) 4370-4379. 


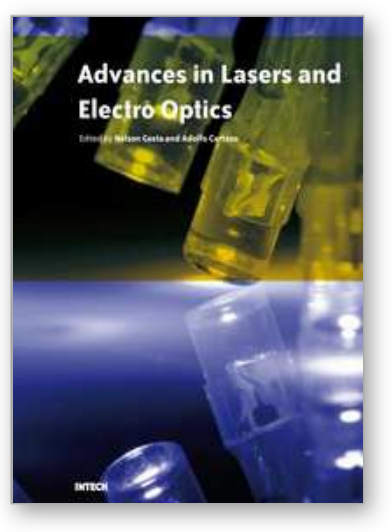

\author{
Advances in Lasers and Electro Optics \\ Edited by Nelson Costa and Adolfo Cartaxo
}

ISBN 978-953-307-088-9

Hard cover, 838 pages

Publisher InTech

Published online 01, April, 2010

Published in print edition April, 2010

Lasers and electro-optics is a field of research leading to constant breakthroughs. Indeed, tremendous advances have occurred in optical components and systems since the invention of laser in the late $50 \mathrm{~s}$, with applications in almost every imaginable field of science including control, astronomy, medicine, communications, measurements, etc. If we focus on lasers, for example, we find applications in quite different areas. We find lasers, for instance, in industry, emitting power level of several tens of kilowatts for welding and cutting; in medical applications, emitting power levels from few milliwatt to tens of Watt for various types of surgeries; and in optical fibre telecommunication systems, emitting power levels of the order of one milliwatt. This book is divided in four sections. The book presents several physical effects and properties of materials used in lasers and electro-optics in the first chapter and, in the three remaining chapters, applications of lasers and electro-optics in three different areas are presented.

\title{
How to reference
}

In order to correctly reference this scholarly work, feel free to copy and paste the following:

Greg Sun (2010). The Intersubband Approach to Si-based Lasers, Advances in Lasers and Electro Optics, Nelson Costa and Adolfo Cartaxo (Ed.), ISBN: 978-953-307-088-9, InTech, Available from:

http://www.intechopen.com/books/advances-in-lasers-and-electro-optics/the-intersubband-approach-to-sibased-lasers

\section{INTECH}

open science | open minds

\section{InTech Europe}

University Campus STeP Ri

Slavka Krautzeka 83/A

51000 Rijeka, Croatia

Phone: +385 (51) 770447

Fax: +385 (51) 686166

www.intechopen.com

\section{InTech China}

Unit 405, Office Block, Hotel Equatorial Shanghai

No.65, Yan An Road (West), Shanghai, 200040, China

中国上海市延安西路65号上海国际贵都大饭店办公楼 405 单元

Phone: +86-21-62489820

Fax: $+86-21-62489821$ 
(C) 2010 The Author(s). Licensee IntechOpen. This chapter is distributed under the terms of the Creative Commons Attribution-NonCommercialShareAlike-3.0 License, which permits use, distribution and reproduction for non-commercial purposes, provided the original is properly cited and derivative works building on this content are distributed under the same license. 